\title{
Water abundance in four of the brightest water sources in the southern sky
}

\author{
Bing-Ru Wang ${ }^{1}$, Lei Qian ${ }^{1}$, Di Li ${ }^{1,2}$ and Zhi-Chen Pan $^{1}$ \\ 1 National Astronomical Observatories, Chinese Academy of Sciences, Beijing 100012, China; brwang@nao.cas.cn \\ 2 Key Laboratory of Radio Astronomy, Chinese Academy of Sciences, Nanjing 210008, China
}

Received 2015 April 3; accepted 2015 September 11

\begin{abstract}
We estimated the ortho- $\mathrm{H}_{2} \mathrm{O}$ abundances of G267.9-1.1, G268.4-0.9, G333.1-0.4 and G336.51.5 , four of the brightest ortho- $\mathrm{H}_{2} \mathrm{O}$ sources in the southern sky observed by the Submillimeter Wave Astronomy Satellite (ortho- $\mathrm{H}_{2} \mathrm{O} 1_{10}-1_{01}$ line, $556.936 \mathrm{GHz}$ ). The typical molecular clumps in our sample have $\mathrm{H}_{2}$ column densities of $10^{22}$ to $10^{23} \mathrm{~cm}^{-2}$ and ortho- $\mathrm{H}_{2} \mathrm{O}$ abundances of $10^{-10}$. Compared with previous studies, the ortho- $\mathrm{H}_{2} \mathrm{O}$ abundances are at a low level, which can be caused by the low temperatures of these clumps. To estimate the ortho- $\mathrm{H}_{2} \mathrm{O}$ abundances, we used the $\mathrm{CS} J=2 \rightarrow 1$ line $(97.98095 \mathrm{GHz})$ and CS $J=5 \rightarrow 4(244.93556 \mathrm{GHz})$ line observed by the Swedish-ESO $15 \mathrm{~m}$ Submillimeter Telescope (SEST) to calculate the temperatures of the clumps and the $350 \mu \mathrm{m}$ dust continuum observed by the Caltech Submillimeter Observatory (CSO) telescope to estimate the $\mathrm{H}_{2}$ column densities. The observations of $\mathrm{N}_{2} \mathrm{H}^{+}$ $(J=1 \rightarrow 0)$ for these clumps were also acquired by SEST and the corresponding abundances were estimated. The $\mathrm{N}_{2} \mathrm{H}^{+}$abundance in each clump shows a common decreasing trend toward the center and a typical abundance range from $10^{-11}$ to $10^{-9}$.
\end{abstract}

Key words: ISM: abundances — (ISM:) HII regions — ISM: molecules — stars: formation

\section{INTRODUCTION}

Water was first detected in the interstellar medium (ISM) over 40 years ago (Cheung et al. 1969). It is an essential coolant in star-forming regions and plays an important role in the energy balance of prestellar objects (Doty \& Neufeld 1997). Thus, the abundance of water is a crucial parameter, especially for massive star formation (Emprechtinger et al. 2010). Since the physical conditions of star-forming regions affect the water abundance (with respect to $\mathrm{H}_{2}$ ), water acts as an excellent diagnostic for energetic phenomena (Kristensen \& van Dishoeck 2011). As an abundant oxygen-bearing molecule formed in molecular clouds, its abundance also gives constraints on the abundance of atomic oxygen, therefore it affects the abundances of other chemically related oxygen-bearing species.

Accessible water lines and feasible methods are necessary for estimating the abundance of water in star-formingregions. Water lines originating from different levels probe gas under different conditions. Most rotational water lines, including the ground-state transition of ortho- and para$\mathrm{H}_{2} \mathrm{O}$, cannot be observed from the ground due to the existence of telluric water (Emprechtinger et al. 2010). Although there are indeed some transitions that have been detected from the ground, their upper states are over $200 \mathrm{~K}$ above the ground state (Snell et al. 2000a). The high energies over the ground state indicate high gas temperatures when collision with $\mathrm{H}_{2}$ is considered as the excitation mechanism. Thus, these transitions are unlikely to be from cold gases. To date, space observations, (e.g., the Submillimeter Wave Astronomy Satellite (SWAS) (Melnick et al. 2000); the Odin satellite; the Infrared Space Observatory (ISO); the Spitzer Space Telescope and the Herschel Space Observatory) have detected water lines, including the $556.936 \mathrm{GHz}$ ortho- $\mathrm{H}_{2} \mathrm{O} 1_{10}-1_{01}$ line. This ground-state transition was observed by SWAS first. With the upper state lying only $27 \mathrm{~K}$ above the ortho- $\mathrm{H}_{2} \mathrm{O}$ ground state, it provides access to estimate the water abundance in cold molecular gas, in which massive stars form in cold dense clumps and young stellar objects are deeply buried.

Water can form in several different routes, both in gas phase and on dust grains. Once they form, the $\mathrm{H}_{2} \mathrm{O}$ molecules can be desorbed from the ice mantle of dusts, remain frozen on the dust surface or freeze onto the dust grains from the gas phase. Water ice on the dust surface can desorb thermally when the dust temperature rises above about $100 \mathrm{~K}$ (Hollenbach et al. 2009). In another way, photodesorption occurs when the ice absorbs ultraviolet (UV) photons (van Dishoeck et al. 2013). When the temperature is as low as about $10 \mathrm{~K}$ and the density is high enough, freeze-out will dominate (Bergin \& van Dishoeck 2012) and consequently lead to low $\mathrm{H}_{2} \mathrm{O}$ abundances. Thus, temperature and UV radiation are essential factors that affect water abundance.

To compare observations with predicted results, the abundances of para- or ortho- $\mathrm{H}_{2} \mathrm{O}$ are estimated based on the spectra obtained from telescopes. For the ortho- 
$\mathrm{H}_{2} \mathrm{O}$ line $\left(1_{10}-1_{01}, 556.936 \mathrm{GHz}\right)$, an effectively optically thin approximation (Snell et al. 2000a) was adopted, which makes it convenient to estimate the ortho- $\mathrm{H}_{2} \mathrm{O}$ abundance. In the Herschel key programme "water in star-forming regions with Hersehel" (WISH), the nonlocal thermodynamic equilibrium (LTE) radiative transfer code RADEX (van der Tak et al. 2007) was used to reduce the ortho- $\mathrm{H}_{2} \mathrm{O}$ line $\left(1_{10}-1_{01}, 556.936 \mathrm{GHz}\right)$ data to estimate the $\mathrm{H}_{2} \mathrm{O}$ abundance in a low-mass protostar Kristensen et al. 2012). In this paper, we estimate the ortho- $\mathrm{H}_{2} \mathrm{O}$ abundances of four of the brightest ortho$\mathrm{H}_{2} \mathrm{O}$ sources (G267.9-1.1, G268.4-0.9, G333.1-0.4 and G336.5-1.5) in the southern sky observed by SWAS. The paper is organized as follows: in Section 2, we briefly describe the observations of these clumps and the data reduction procedures. In Section 3, we present the calculations and estimates of clump temperatures, clump masses, $\mathrm{H}_{2}$ column densities and finally the estimate of ortho$\mathrm{H}_{2} \mathrm{O}$ abundances based on observations. In Section 4 and Section 5, we present the discussion and conclusion respectively. The appendix contains some supplementary material.

\section{OBSERVATION AND DATA REDUCTION}

\subsection{Source Selection}

We checked the co-added spectra of the ortho- $\mathrm{H}_{2} \mathrm{O} 1_{10}-$ $1_{01}$ line of all the 386 sources in the five and a half years of the SWAS nominal mission from Lambda1. We selected four of the sources with $T_{\mathrm{A}}^{*}$ higher than $0.1 \mathrm{~K}$ (excluding the sources in the Galactic Center region) in the southern sky. These four sources are G267.9-1.1 $\left(T_{\mathrm{A}}^{*}=0.10 \mathrm{~K}\right)$, G268.4-0.9 $\left(T_{\mathrm{A}}^{*}=0.16 \mathrm{~K}\right), \mathrm{G} 333.1-0.4\left(T_{\mathrm{A}}^{*}=0.20 \mathrm{~K}\right)$ and $\mathrm{G} 336.5-1.5\left(T_{\mathrm{A}}^{*}=0.45 \mathrm{~K}\right)$. Among these four sources, G336.5-1.5 has the highest $T_{\mathrm{A}}^{*}$.

These four sources are located in star forming regions RCW 38 (G267.9-1.1 and G268.4-0.9), RCW 106 (G333.1-0.4) and RCW 108 (G336.5-1.5), respectively. Although being bright at $8 \mu \mathrm{m}$, they are all associated with the $22 \mathrm{GHz} 66_{16}-5_{23}$ water masers (Kaufmann et al. 1976; Braz et al. 1989; Caswell et al. 1974 and Valdettaro et al. 2007), which are believed to be good indicators of the location of massive star formation (Juvela 1996). The properties of these four sources are summarized briefly as follows.

(1) G267.9-1.1. It is the third brightest source in the investigation of Galactic radio sources at $5000 \mathrm{MHz}$ (Goss \& Shaver 1970), with a brightness temperature of $124.0 \mathrm{~K}$ (Shaver \& Goss 1970). The associated $22 \mathrm{GHz} \quad 6_{16} \quad-5_{23}$ water maser (without $\mathrm{OH}$ mainline emission) was first reported by Kaufmann et al. (1976).

(2) G268.4-0.9. It was identified in an $11 \mathrm{~cm}$ survey of Vela (Manchester \& Goss 1969), near the source

\footnotetext{
1 http://lambda.gsfc.nasa.gov/product/swas/s_sw.cfm
}

G267.9-1.1 (denoted as G268.0-1.0 in the same survey) with a lower brightness temperature. However, it was not identified as an isolated radio source in the Galactic radio source survey (Goss \& Shaver 1970). The associated $22 \mathrm{GHz}$ water maser was identified by Braz et al. (1989).

(3) G333.1-0.4. It is one of the clumps in the giant molecular cloud (GMC) G333 (Lowe et al. 2014). It was first identified as an extensive HII region (Beard 1966), with a brightness temperature of $17.8 \mathrm{~K}$ (Shaver \& Goss 1970). The associated $22 \mathrm{GHz}$ water maser was identified by Caswell et al. (1974). The ortho- $\mathrm{H}_{2} \mathrm{O} 556.936 \mathrm{GHz}$ line obtained by the SWAS exhibits a pronounced inverse P Cygni profile and related study ( $\mathrm{Li}$ et al.2004) suggests that it is a rare case of direct observational evidence for large scale infall in a star forming region.

(4) G336.5-1.5. It is identified as an isolated compact HII region in both the survey of $\mathrm{H} 109 \alpha$ recombination line emission in Galactic HII regions of the southern sky Wilson et al. 1970) and the investigation of Galactic radio sources at $5000 \mathrm{MHz}$ (Goss \& Shaver 1970). Its brightness temperature is $7.2 \mathrm{~K}$ (Shaver \& Goss 1970). G336.5-1.5 is associated with bright-rimmed cloud (BRC) 79, one of the 89 clouds in a catalog of BRCs with IRAS point sources Sugitani \& Ogura 1994). It has the largest $\mathrm{H}_{2}$ column density among the 43 southern hemisphere BRCs (BRC 77 and BRC 78 excluded) and the HII region RCW 62, according to ${ }^{13} \mathrm{CO}$ observations (Yamaguchi et al. 1999). Its ortho- $\mathrm{H}_{2} \mathrm{O} 556.936 \mathrm{GHz}$ line obtained by SWAS exhibits the highest antenna temperature among all observed sources (other than solar system objects and the Galactic Center), which makes it an interesting object to study.

Compared with the other three sources, G336.5-1.5 has a higher Galactic latitude. Its associated $22 \mathrm{GHz}$ water maser was detected in a survey of 45 southern BRCs (Sugitani \& Ogura 1994) for $\mathrm{H}_{2} \mathrm{O}$ maser emission Valdettaro et al. 2007), with a total integrated $\mathrm{H}_{2} \mathrm{O}$ flux density of merely $5.4 \mathrm{Jy} \mathrm{km} \mathrm{s}^{-1}$. All these features mentioned above imply that these four sources are likely to be massive star forming active clumps. We use "clumps" to refer to these four sources in this paper.

\subsection{Observation and Data Reduction}

The observations were carried out with three telescopes. The ortho- $\mathrm{H}_{2} \mathrm{O} 1_{10}-1_{01}$ line $(556.936 \mathrm{GHz})$ was observed with SWAS. The CS $J=2 \rightarrow 1$ line (97.98095 GHz), CS $J=5 \rightarrow 4$ line $(244.93556 \mathrm{GHz})$ and $\mathrm{N}_{2} \mathrm{H}^{+} J=1 \rightarrow 0$ line $(93.17340 \mathrm{GHz})$ data were from the Swedish-ESO $15 \mathrm{~m}$ Submillimeter Telescope (SEST2). The $350 \mu \mathrm{m}$ dust continuum data were obtained with the Submillimeter High Angular Resolution

\footnotetext{
2 http://www.eso.org/public/images/esopia00049teles/
} 
Camera II (SHARC II; see Dowell et al. 2003) of the Caltech Submillimeter Observatory (CSO) telescope. The observational parameters of molecular lines are listed in Table 1 .

\subsubsection{SWAS observation}

The observations of the ortho- $\mathrm{H}_{2} \mathrm{O} 1_{10}-1_{01}$ line $(556.936 \mathrm{GHz})$ were performed with SWAS from 1999 January 20 to 2001 May 3 (G267.9-1.1), 1998 December 20 to 2003 June 5 (G268.4-0.9), 1999 September 15 to 2002 February 25 (G333.1-0.4) and 2001 September 22 to 2004 July 21 (G336.5-1.5). The data were obtained from the SWAS spectrum service in the NASA/IPAC infrared science archive 3 .

The ortho- $\mathrm{H}_{2} \mathrm{O} 557 \mathrm{GHz} 1_{10}-1_{01}$ line data acquired by SWAS were converted into FITS format with a uniform $190 \times 190 \operatorname{arcsec}^{2}$ pixel size after the spectra in every single beam (which are also in the same sampling cell) were averaged and then the baselines were substracted. The Gildas software packaget was used for averaging and baseline subtraction. The baselines of spectra were acceptable and a $1^{\text {st }}$ or $2^{\text {nd }}$ order polynomial was used for baseline fitting. The typical root mean squares (RMSs) of the ortho- $\mathrm{H}_{2} \mathrm{O} 557 \mathrm{GHz} 1_{10}-1_{01}$ spectra are $0.017 \mathrm{~K}$ for G267.9-1.1, 0.013 K for G268.4-0.9, 0.014 K for G333.10.4 , and $0.03 \mathrm{~K}$ for G336.5-1.5. The different RMSs are mainly due to different integration times. When we calculated the integrated intensities of the ortho- $\mathrm{H}_{2} \mathrm{O} 557 \mathrm{GHz}$ $1_{10}-1_{01}$ line, the antenna temperatures were corrected with a main beam efficiency of 0.9 .

For G268.4-0.9 and G333.1-0.4, the antenna temperatures below zero are due to the high noises and the subtraction of the baseline. The double-peaked spectra of ortho$\mathrm{H}_{2} \mathrm{O} 1_{10}-1_{01}$ lines of G268.4-0.9 indicated strong selfabsorption (Ashby et al. 2000). We performed a Gaussian fitting for both non-absorbed emission peaks and the absorption peaks and obtained the integrated intensity of the emission of the averaged and baseline subtracted spectrum. The spectrum of G333.1-0.4 shows a pronounced inverse $P$ Cygni profile. We took into account both the water components corresponding to emission and absorption features.

\subsubsection{SEST observation}

The observations of the CS $J=2 \rightarrow 1$ line (97.98095 GHz), CS $J=5 \rightarrow 4$ line $(244.93556 \mathrm{GHz})$ and $\mathrm{N}_{2} \mathrm{H}^{+} J=1 \rightarrow 0$ line $(93.17632 \mathrm{GHz})$ were carried out with SEST. These four clumps were mapped with CS $J=2 \rightarrow 1$ (except for G333.1-0.4), CS $J=5 \rightarrow 4$ and $\mathrm{N}_{2} \mathrm{H}^{+} J=1 \rightarrow 0$ in 2002 Mar 24-28. The mainbeam efficiencies were $0.73(\mathrm{CS} J=2 \rightarrow 1$ ), 0.56 $(\mathrm{CS} J=5 \rightarrow 4)$ (Lapinov et al. 1998) and $0.74\left(\mathrm{~N}_{2} \mathrm{H}^{+}\right.$ $J=1 \rightarrow 0$ ) (Mardones et al. 1997), respectively.

3 http://irsa.ipac.caltech.edu/applications/SWAS/SWAS/list.html

4 http://www.iram.fr/IRAMFR/GILDAS/
In the mappings, the spacing of the square scanning grids is $40^{\prime \prime}$, but in the CS (5-4) mappings for G268.4-0.9, G333.1-0.4 and G336.5-1.5, additional sampling made the square scanning grids quincunxes. In each map, every pixel was observed with the position switch mode separately. The reference positions are selected approximately $1800^{\prime \prime}$ away from the centers of the maps (the coordinates in Columns (2) and (3) of Table 3).

The Gildas software package was also used. For several spectra, the baselines seem to follow a sine function but with changing periods and amplitudes. In addition, for several spectra the line widths of the real emission lines are similar to the periods of their sine baselines, so we left them with their sinusoidal baselines. We check all the spectra one by one for baseline fitting. These spectra are near the edge of the mapping area and our calculation results are affected little.

After the baseline subtraction, the obtained spectra with RMSs less than $1 \mathrm{~K}$ (for CS $J=5 \rightarrow 4$ in G333.10.4 and $\mathrm{G} 336.5-1.5$, the sigma limits are $0.5 \mathrm{~K}$ and $0.84 \mathrm{~K}$, respectively) were selected and written in FITS format with a uniform $40 \times 40 \operatorname{arcsec}^{2}$ pixel size.

The RMSs of the CS (2-1) spectra in the center of the images are 0.16 K for G267.9-1.1 (RA = 08:59:12.0, Dec $=-47: 29: 04), 0.16 \mathrm{~K}$ for $\mathrm{G} 268.4-0.9(\mathrm{RA}=09: 01: 54.3$, Dec $=-47: 43: 59)$ and $0.16 \mathrm{~K}$ for $\mathrm{G} 336.5-1.5(\mathrm{RA}=$ 16:39:58.9, Dec $=-48: 51: 00)$. The RMSs of the CS (5-4) spectra in the center of the images are $0.42 \mathrm{~K}$ for G267.9$1.1(\mathrm{RA}=08: 59: 12.0, \mathrm{Dec}=-47: 29: 04), 0.16 \mathrm{~K}$ for G268.4-0.9 (RA = 09:01:54.3, Dec $=-47: 43: 59), 0.13 \mathrm{~K}$ for $\mathrm{G} 333.1-0.4(\mathrm{RA}=16: 21: 02.1, \mathrm{Dec}=-50: 35: 15)$, and $0.09 \mathrm{~K}$ for $\mathrm{G} 336.5-1.5(\mathrm{RA}=16: 39: 58.9$, Dec $=$ $-48: 51: 00)$.

We did not have CS (2-1) data for G333.1-0.4. The RMSs of the $\mathrm{N}_{2} \mathrm{H}^{+}(1-0)$ spectra in the center of the images are $0.20 \mathrm{~K}$ for $\mathrm{G} 267.9-1.1(\mathrm{RA}=08: 59: 12.0$, Dec $=$ $-47: 29: 04), 0.18 \mathrm{~K}$ for G268.4-0.9 (RA = 09:01:54.3, Dec $=-47: 43: 59), 0.19 \mathrm{~K}$ for G333.1-0.4 $(\mathrm{RA}=16: 21: 00.8$, Dec $=-50: 34: 55)$, and $0.17 \mathrm{~K}$ for G336.5-1.5 (RA = 16:39:58.9, Dec $=-48: 51: 00)$.

\subsubsection{CSO observation}

The $350 \mu \mathrm{m}$ dust continuum observations were performed with SHARC II on CSO during 2014 April 4 and 5. The data were taken when these four regions were close to their maximum elevation (approximately 20 degrees at the CSO site) and the $\tau_{225 \mathrm{GHz}}$ was lower than 0.06 . The box scan mode was used for the SHARC II observation. The beam size of SHARC II is $8^{\prime \prime}$ and the grid spacing for the sampling is $1.5 \times 1.5 \operatorname{arcsec}^{2}$. For each scan, the total integration time is 14.71 minutes and the corresponding RMS is 212 mJy beam $^{-1}$. Pointing and focusing calibration was done every 2 hours during the observation. The data reduction tool CRUSH 5 was used for further data reduction. The flux calibration was done by observing Mars.

\footnotetext{
5 http://www.submm.caltech.edu/ sharc/crush/
} 
Table 1 Observational Parameters of Molecular

Lines

\begin{tabular}{lccccc}
\hline Transition & $\begin{array}{c}\text { Frequency } \\
(\mathrm{GHz})\end{array}$ & Instrument & Beam Size & $\begin{array}{c}\Delta \nu \\
(\mathrm{kHz})\end{array}$ & $\begin{array}{c}\Delta v \\
\left(\mathrm{~km} \mathrm{~s}^{-1}\right)\end{array}$ \\
\hline Ortho- $\mathrm{H}_{2} \mathrm{O} 1_{10-101}$ & 556.93599 & SWAS & $3.3^{\prime} \times 4.5^{\prime a}$ & $1.0 \times 10^{3}$ & 0.55 \\
$\mathrm{CS}(2-1)$ & 97.98095 & SEST & $42^{\prime \prime}$ & 43 & 0.13 \\
$\mathrm{CS}(5-4)$ & 244.93556 & SEST & $17^{\prime \prime}$ & $43^{b}$ & $0.052^{b}$ \\
$\mathrm{~N}_{2} \mathrm{H}^{+}(1-0)$ & 93.17304 & SEST & $44^{\prime \prime}$ & 43 & 0.14 \\
\hline
\end{tabular}

Notes: ${ }^{a}$ Melnick et al. 2000; ${ }^{b}$ For G267.9-1.1, $\Delta v$ of the CS (5-4) line is $0.060 \mathrm{~km} \mathrm{~s}^{-1}$, and $\Delta \nu$ is $49 \mathrm{kHz}$.

The RMS for the final data are $0.49 \mathrm{Jy} \mathrm{beam}^{-1}$ for G267.9$1.1,0.61 \mathrm{Jy}^{\text {beam }^{-1}}$ for G268.4-0.9, $0.79 \mathrm{Jy} \mathrm{beam}^{-1}$ for G333.1-0.4 and $0.53 \mathrm{Jy}_{\text {beam }}{ }^{-1}$ for G336.5-1.5. The weather is the main reason for the variation of the noises in different maps.

\section{RESULTS AND ANALYSIS}

\subsection{Spectra Map and Dust Map}

Figures 1, 2, 3 and 4 are line profile maps of G267.91.1, G268.4-0.9, G333.1-0.4 and G333.6-1.5. In these line profile maps, all offsets are relative to the corresponding coordinates (see Table 3) and the units are arcsec. Empty boxes are the positions without sampling.

There is a "hole" with little CS (2-1 and 5-4) emission in the center of the emission region of G267.9-1.1. Moreover, the centroid velocities of the CS spectra in the east of the hole are different from those of the CS spectra in the west of the hole. In the south of the hole, the CS spectra all have two obvious peaks and in the north of the hole, the spectra all have two peaks as well. We overlapped the CS (2-1) integrated intensity map on the $350 \mu \mathrm{m}$ dust continuum in Figure 5 We can see that the intensity peaks are associated with the $350 \mu \mathrm{m}$ emission and in the "hole" the dust emission is much weaker than the surrounding areas.

The RMSs of CS (5-4) spectra of G333.1-0.4 vary a lot, which is caused by different integration times. The integration time (on source time) changes from less than 0.8 minutes to more than 3 minutes. In G336.5-1.5's CS (5-4) spectra, there are similar situations.

The $350 \mu \mathrm{m}$ dust continuums of these four clumps are shown in Figure 6 In the following sections, we estimated the temperatures, masses, $\mathrm{H}_{2}$ column densities and orthowater abundances of these four clumps. The areas for mass and ortho-water abundance estimates are shown in white boxes with solid lines and dashed lines, in the corresponding figures, respectively.

\subsection{CS Excitation Temperatures}

\subsubsection{The estimate of CS excitation temperatures}

For G267.9-1.1, G268.4-0.9 and G336.5-1.5 (we did not have CS (2-1) line data for G333.1-0.4) the excitation temperatures of the CS molecule were estimated based on the CS (2-1) line and CS (5-4) line. The estimated CS exci- tation temperatures were subsequently adopted in the estimates of clump masses (and then the $\mathrm{H}_{2}$ column densities), ortho-water abundances and $\mathrm{N}_{2} \mathrm{H}^{+}$abundances.

The estimate is based on the following assumptions, i.e., (1) The CS molecules are in LTE. (2) The cosmic microwave background radiation $(\mathrm{CMB})$ can be ignored. (3) CS (2-1) and CS (5-4) lines are optically thin. Since G267.9-1.1, G268.40.9 and G336.5-1.5 all fill the main beam, the filling factors in the estimate equal 1 . According to the population diagram method (in LTE) (Goldsmith \& Langer 1999), for the upper levels $J=2$ and $J=5$ there are

$$
\ln \frac{N_{\mathrm{J}=2}^{\mathrm{thin}}}{g_{\mathrm{J}=2}}+\ln \frac{\tau_{2-1}}{1-e^{\tau_{2-1}}}=\ln N_{\text {tot }}-\ln Z-\frac{E_{\mathrm{J}=2}}{k T_{\mathrm{ex}}},
$$

and

$$
\ln \frac{N_{\mathrm{J}=5}^{\mathrm{thin}}}{g_{\mathrm{J}=5}}+\ln \frac{\tau_{5-4}}{1-e^{\tau_{5-4}}}=\ln N_{\mathrm{tot}}-\ln Z-\frac{E_{\mathrm{J}=5}}{k T_{\mathrm{ex}}},
$$

respectively. $N_{\mathrm{J}=2}^{\text {thin }}$ and $N_{\mathrm{J}=5}^{\text {thin }}$ are the column densities at $J=2$ and $J=5$ in the optically thin situation respectively. $g_{\mathrm{J}=2}$ and $g_{\mathrm{J}=5}$ are the statistical weights of level $J=2$ and level $J=5$ respectively, and $\tau_{5-4}$ and $\tau_{2-1}$ are the corresponding optical depths. $N_{\text {tot }}$ is the total column density of the CS molecule and $Z$ is the partition function. $T_{\text {ex }}$ is the excitation temperature of these two transitions. Since we assumed that the CS (2-1) line and CS (5-4) line are optically thin, we considered

$$
\ln \frac{\tau_{2-1}}{1-e^{\tau_{2-1}}}=0
$$

and

$$
\ln \frac{\tau_{5-4}}{1-e^{\tau_{5-4}}}=0 .
$$

From Equations (1), (2), (3) and (4), we obtained

$$
T_{\mathrm{ex}}=\frac{E_{\mathrm{J}=5}-E_{\mathrm{J}=2}}{k \ln \left(\frac{g_{\mathrm{J}=5} N_{\mathrm{J}=2}^{\text {thin }}}{g_{\mathrm{J}=2} N_{\mathrm{J}=5}^{\text {thin }}}\right)},
$$

while

$$
E_{J}=h B_{\mathrm{e}} J(J+1) .
$$

$J$ is the rotational quantum number and $B_{\mathrm{e}}$ is the rotational constant of the CS molecule at vibrational energy level $v$ $=0$ in $\mathrm{Hz}\left(2.458437 \times 10^{10} \mathrm{~Hz}\right.$, Kewley et al. 1963). The statistical weights of the $J=2$ and $J=5$ level, $g_{\mathrm{J}=2}$ 

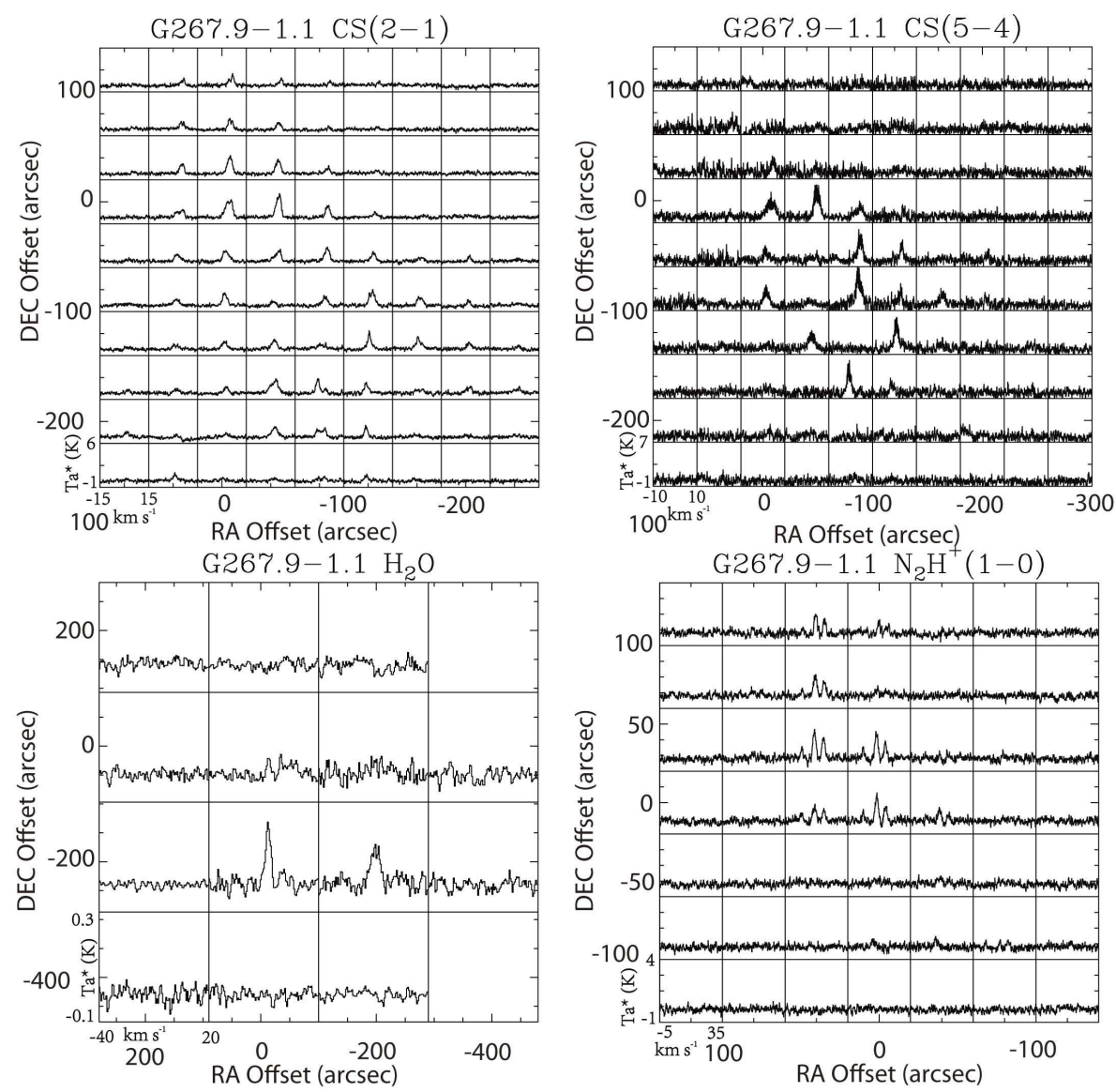

Fig. 1 Upper Left: G267.9-1.1 CS (2-1) line profile map. It is obvious that at positions $\left(-40^{\prime \prime},-80^{\prime \prime}\right)$ and $\left(-80^{\prime \prime},-120^{\prime \prime}\right)$ relative to the coordinates in Table 3, the emissions at these two positions are much weaker than the surroundings and that may suggest "holes" in molecular gas with significantly lower $\mathrm{H}_{2}$ volume density. Similar and more significant phenomena can be seen in the CS (5-4) line profile map. Upper Right: G267.9-1.1 CS (5-4) line profile map. Lower Left: G267.9-1.1 ortho- $\mathrm{H}_{2} \mathrm{O} 1_{10}-1_{01}$ line profile map. The two empty boxes are positions without sampling. Lower Right: G267.9-1.1 $\mathrm{N}_{2} \mathrm{H}^{+}(1-0)$ line profile map.

and $g_{\mathrm{J}=5}$, equal 5 and 11 respectively. Thus, we can write Equation (5) as

$$
T_{\mathrm{ex}}=\frac{24 h B_{\mathrm{e}}}{k \ln \left(\frac{11 N_{\mathrm{J}}^{\text {thin }}}{5 N_{\mathrm{J}=5}^{\text {thin }}}\right)} .
$$

Now we focus on the CS column densities of the upper levels $(J=2$ and $J=5$ ). Based on Rohlfs \& Wilson (1996), when the molecular line is optically thin, the column densities of the upper level $(J=2$ or $J=5)$ are

$$
N_{\mathrm{u}}^{\mathrm{thin}}=\frac{8 \pi \nu^{3}}{c^{3} A_{\mathrm{ul}}\left(e^{\frac{h \nu}{k T_{\mathrm{ex}}}}-1\right)} \int \tau_{\nu} d v,
$$

where $\nu$ is the frequency of the CS (2-1) or CS (5-4) line, $T_{\text {ex }}$ is the excitation temperature, $A_{\mathrm{ul}}$ is the corresponding Einstein $A$-coefficient and $\tau_{\nu}$ is the optical depth. In an isothermal medium, the relationship between the brightness temperature $T_{\mathrm{B}}$, the excitation temperature $T_{\mathrm{ex}}$ and the cosmic background temperature $T_{\text {background }}$ can be described as

$$
T_{\mathrm{B}}=T_{\text {background }} e^{-\tau_{\nu}}+T_{\mathrm{ex}}\left(1-e^{\tau_{\nu}}\right) .
$$

When the CS lines are optically thin,

$$
1-e^{-\tau_{\nu}} \approx \tau_{\nu}
$$

and if the background radiation can be ignored, then

$$
T_{\mathrm{B}} \approx T_{\mathrm{ex}} \tau_{\nu}
$$

Substituting Equation (11) into Equation (8), we obtained

$$
\begin{aligned}
N_{\mathrm{u}}^{\mathrm{thin}} & =\frac{8 \pi \nu^{3}}{c^{3} A_{\mathrm{ul}} T_{\mathrm{ex}}\left(e^{\frac{h \nu}{k T_{\mathrm{ex}}}}-1\right)} \int T_{\mathrm{ex}} \tau_{\nu} d v \\
& =\frac{8 \pi \nu^{3}}{c^{3} A_{\mathrm{ul}} T_{\mathrm{ex}}\left(e^{\frac{h \nu}{k T_{\mathrm{ex}}}}-1\right)} \int T_{\mathrm{B}} d v,
\end{aligned}
$$

Since the four clumps in our study are all extended sources, the antenna temperature $T_{\mathrm{a}} \simeq T_{\mathrm{B}}$, and the column densities of upper levels in the optically thin case are

$$
N_{\mathrm{u}}^{\mathrm{thin}}=\frac{8 \pi \nu^{3}}{c^{3} A_{\mathrm{ul}} T_{\mathrm{ex}}\left(e^{\frac{h \nu}{k T_{\mathrm{ex}}}}-1\right)} \int T_{\mathrm{a}} d v .
$$



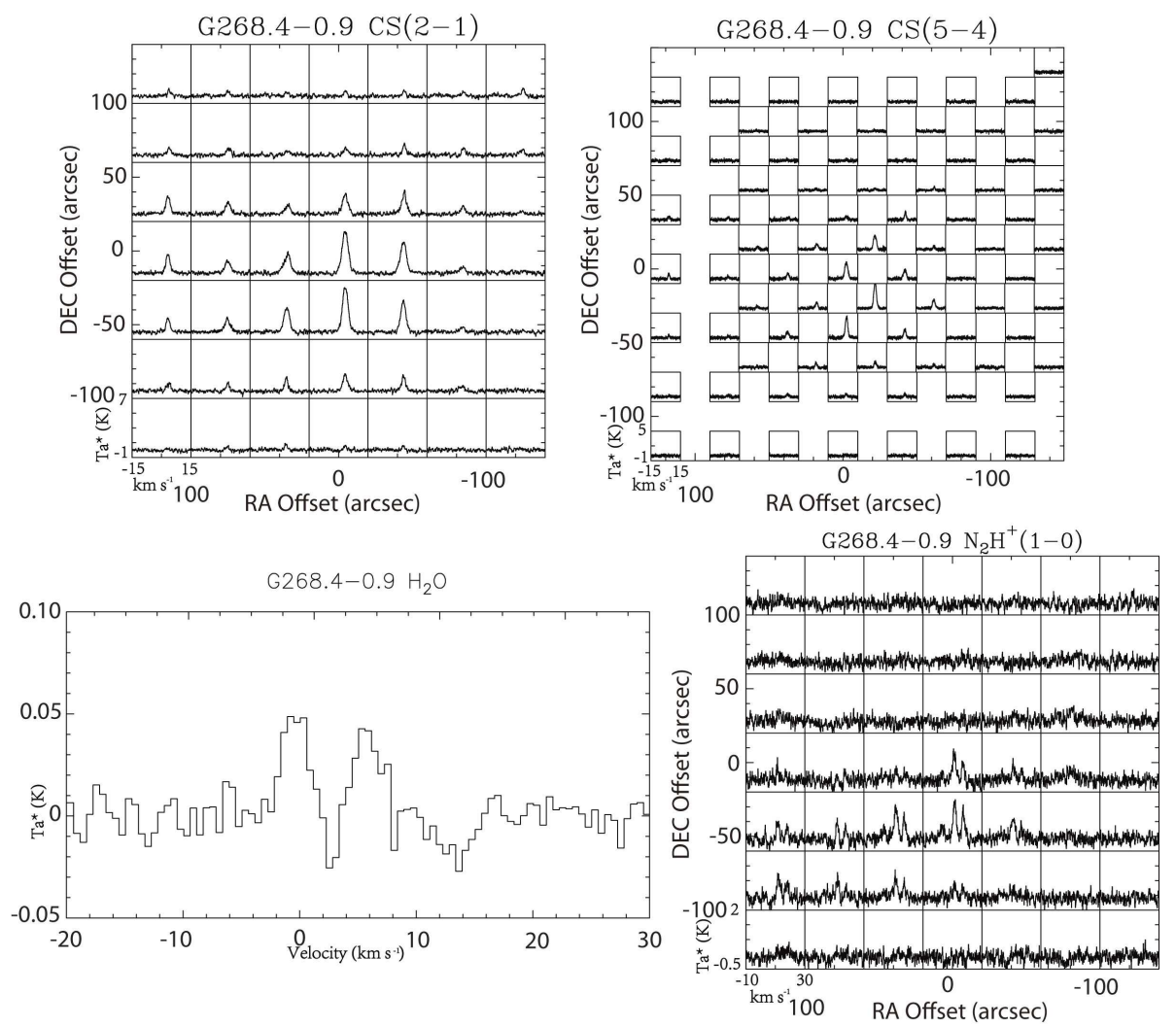

Fig. 2 Upper Left: G268.4-0.9 CS (2-1) line profile map. Upper Right: G268.4-0.9 CS (5-4) line profile map. The empty boxes are positions without sampling. Lower Left: G268.4-0.9 ortho- $\mathrm{H}_{2} \mathrm{O} 1_{10}-1_{01}$ spectrum at RA 09:01:54.31, Dec -47:43:59.0. Lower Right: G268.4-0.9 $\mathrm{N}_{2} \mathrm{H}^{+}$(1-0) line profile map.

In Equation (13), $N_{\mathrm{u}}^{\text {thin }}$ is dependent on $T_{\text {ex }}$.

If we adopt the Rayleigh-Jeans approximation

$$
h \nu \ll k T_{\text {ex }}
$$

in Equation (13), then this equation will be reduced to

$$
N_{\mathrm{u}}^{\mathrm{thin} *}=\frac{8 \pi k \nu^{2}}{h c^{3} A_{\mathrm{ul}}} \int T_{\mathrm{a}} d v .
$$

This expression is the same as that from Goldsmith \& Langer (1999), and it does not depend on the excitation temperature $T_{\mathrm{ex}}$.

However, we notice that if we estimate the column densities of upper levels through Equation (15), then significant deviation will arise due to the high frequency of the CS (5-4) line. The deviation in upper level column densities will lead to significant deviation of the subsequently derived $T_{\text {ex }}$.

The excitation-temperature-dependent $N_{\mathrm{u}}^{\text {thin }}$ was derived by correcting the $N_{\mathrm{u}}^{\mathrm{thin} *}$ with line frequency $\nu$ and excitation temperature $T_{\mathrm{ex}}$

$$
N_{\mathrm{u}}^{\mathrm{thin}}=\frac{h \nu}{k T_{\mathrm{ex}}\left(e^{\frac{h \nu}{k T_{\mathrm{ex}}}}-1\right)} \cdot N_{\mathrm{u}}^{\mathrm{thin} *} .
$$

For each sampling position, we need $N_{\mathrm{u}}^{\text {thin }}$ at the $J=2$ and $J=5$ level to calculate the excitation temperature of $\mathrm{CS}$, while the excitation temperature of the CS molecule is required when we derived $N_{\mathrm{u}}^{\text {thin }}$ (Eq. (16)). As a start, we calculated $N_{\mathrm{u}}^{\text {thin* }}$ for the $J=2$ and $J=5$ level (Eq. (15) and then we derived an excitation temperature (Eq. (7)) from $N_{\mathrm{u}}^{\mathrm{thin} *}(J=2$ and $J=5$ level). In the next step, we obtained the first $N_{\mathrm{u}}^{\text {thin }}$ through Equation (16), with which we subsequently calculated an excitation temperature again (Eq. (7)). Since $N_{\mathrm{u}}^{\mathrm{thin}}$ is dependent on $T_{\text {ex }}$, and $T_{\text {ex }}$ is derived from $N_{\mathrm{u}}^{\text {thin }} \mathrm{s}(J=2$ and $J=5$ level $)$, we performed iterative calculations to correct $T_{\text {ex }}$ gradually, i.e., we ran the iteration cycles of " $N_{\mathrm{u}}^{\text {thin }}-T_{\text {ex }}$." We kept comparing the latest $T_{\text {ex }}$ calculated from the latest $N_{\mathrm{u}}^{\mathrm{thin}} \mathrm{s}$ at the $J=2$ and $J=5$ level with the previously calculated $T_{\text {ex }}$. The iterations would not end until the absolute value of the difference between these two $T_{\text {ex }}$ values was less than $0.05 \mathrm{~K}$.

The calculation of CS excitation temperatures was only applied to the positions where both the CS (2-1) and CS (5-4) line intensities were higher than $3 \sigma$. At these positions, gas along the line of sight would be considered CS (5-4)-traced dense gas and the areas corresponding to all these positions are treated as "CS (5-4)-traced areas," in which the CS molecules can be well-excited through collisions (at least for the CS $J=1 \rightarrow 2$ excitation, since the critical density of the CS (5-4) line is far greater than that of the CS (2-1) line) and the calculated CS excitation temperatures approximately equal the kinetic temperatures of the gas; the kinetic temperature of the gas is an 

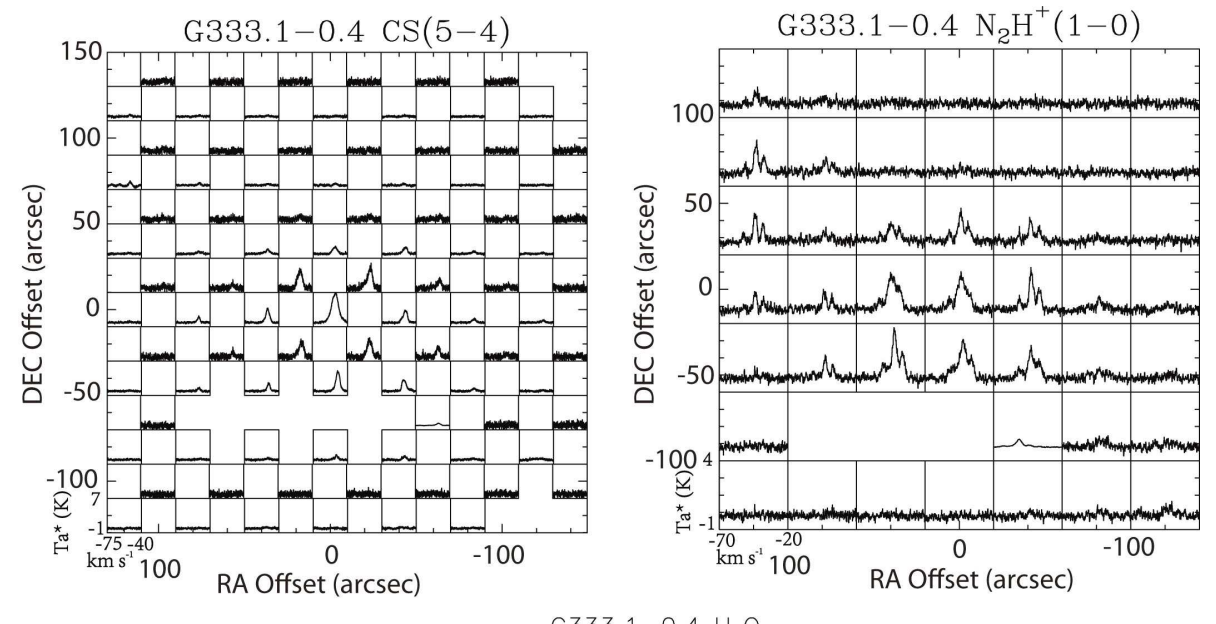

G333.1-0.4 $\mathrm{H}_{2} \mathrm{O}$

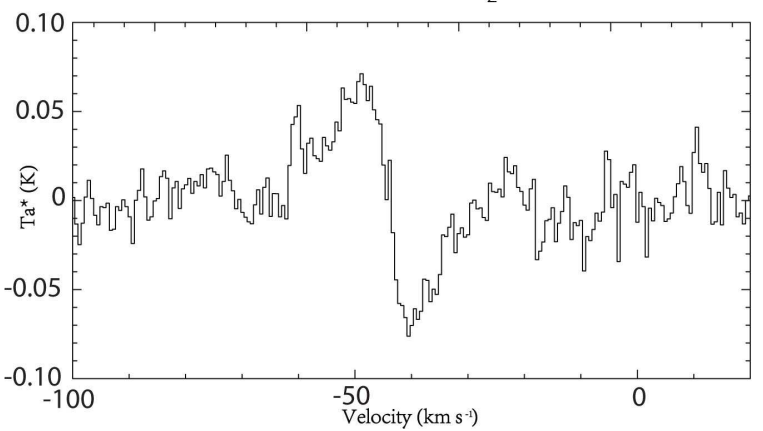

Fig. 3 Upper Left: G333.1-0.4 CS (5-4) line profile map. The empty boxes are positions without sampling. The RMS changes a lot in different spectra. Upper Right: G333.1-0.4 $\mathrm{N}_{2} \mathrm{H}^{+}(1-0)$ line profile map. A spectrum with extremely low RMS is shown. The three empty boxes are positions without sampling. Lower: G333.1-0.4 ortho- $\mathrm{H}_{2} \mathrm{O} 1_{10}-1_{01}$ spectrum at RA 16:21:02.08, Dec-50:35:15.0.

essential parameter in subsequent estimates. Considering the sampling spacing (comparing it with the beam size), no interpolation was applied to positions where the quality of signal is poor (low signal-to-noise ratio) and the corresponding CS column densities at $J=2$ and $J=5$ as well as the $T_{\text {ex }} \mathrm{s}$ were denoted as zero at these positions. The average CS excitation temperatures of these four clumps (corresponding to the areas within the white boxes (solid lines) in Fig. 6) are listed in Column (5) of Table 3 In those areas all calculated CS excitation temperatures are non-zero. We subsequently estimated the masses of the clumps within these white boxes (solid lines) in Section 3.3.

We also derived a "characteristic" CS excitation temperature, $T_{\text {ex-clump }}$, for the CS (5-4)-traced area in each clump (G267.9-1.1, G268.4-0.9 and G336.5-1.5). For each clump, we averaged the integrated intensity of the CS (2-1) line (and CS (5-4) line, as well) at every sample position in the CS (5-4)-traced area (the white boxes (solid lines) in Fig. 6), then with these average integrated intensities we derived the "characteristic" excitation temperatures $T_{\text {ex-clump }}$ through exactly the same assumptions and processes as we previously described.

Figure 7 shows the population diagrams calculated for the $T_{\text {ex-clump }}$ s of G267.9-1.1, G268.4-0.9 and G336.51.5. The population diagrams were deduced from corresponding $N_{\mathrm{u}}^{\text {thin* }}$ s (at $J=2$ and $J=5$, in dashed lines) and the last $N_{\mathrm{u}}^{\mathrm{thin}} \mathrm{s}$ (at $J=2$ and $J=5$, in solid lines) we retrieved in the iteration. We can see that the correction of the upper level column densities $(J=2$ and $J=5)$ for the Rayleigh-Jeans approximation does make a difference in the slopes of these plots. We derived the final values for the $T_{\text {ex-clump }}$ s, i.e., those deduced from the slopes of the plots in solid lines in Figure 7 , were listed in Table 2, together with $N_{\mathrm{CS}-\text { clump }}$, the average CS column density in the CS (5-4)-traced area of each clump. The average CS column density in the CS (5-4)-traced area was deduced from the final results of the iteration for $T_{\mathrm{ex}-\mathrm{clump}}$.

\subsubsection{The non-LTE analysis for CS (2-1) and CS (5-4) lines}

Since it is possible for the CS lines to be optically thick (especially for the CS (2-1) line) in the actual situation, we also performed non-LTE analysis for the CS (2-1) and CS (5-4) lines with RADEX (van der Tak et al. 2007) in the CS (5-4)-traced areas (the white boxes with solid lines in Fig. 6. To perform this analysis, for each clump we added up the integrated intensities of the CS (2-1) line and CS (5-4) line at every sample position in the CS (5-4)traced area (the white boxes (solid lines) in Fig. 6) respectively and found the corresponding average values. Based on the average CS (2-1) and CS (5-4) integrated intensities 

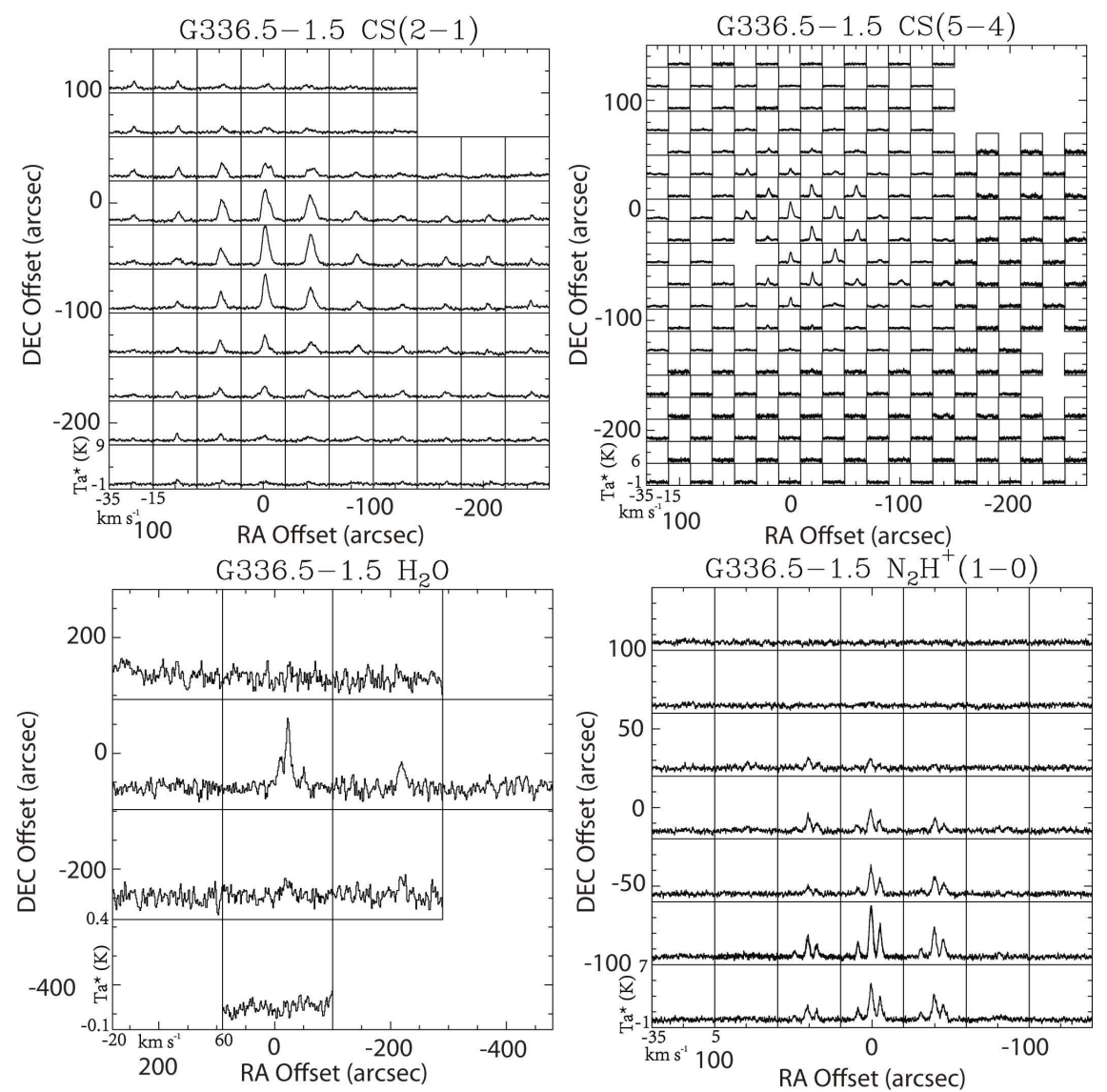

Fig. 4 Upper Left: G336.5-1.5 CS (2-1) line profile map. The empty boxes at the upper right corner are positions without sampling. Upper Right: G336.5-1.5 CS (5-4) line profile map. The empty boxes are positions without an observation. Lower Left: G336.5-1.5 ortho- $\mathrm{H}_{2} \mathrm{O} 1_{10}-1_{01}$ line profile map. The empty boxes are positions without sampling. Lower Right: G336.5-1.5 $\mathrm{N}_{2} \mathrm{H}^{+}$(1-0) line profile map.

we derived the line intensity ratio $R_{(2-1) /(5-4)}$ (CS (21)/CS (5-4)) in the CS (5-4)-traced area for each clump. We then performed the non-LTE analysis with RADEX in a kinetic temperature range from $10 \mathrm{~K}$ to $200 \mathrm{~K}$ and an $\mathrm{H}_{2}$ density range from $10^{3}$ to $10^{8} \mathrm{~cm}^{-3}$. According to the $N_{\text {CS-clump }}$ we had estimated for each clump (see Section 3.2.1), we performed the analysis with CS column densities of $10^{13} \mathrm{~cm}^{-2}$ and $10^{14} \mathrm{~cm}^{-2}$ and got the line intensity ratio maps on the kinetic temperature $\left(T_{\text {kin }}\right)$-density plane. To estimate the probable $\mathrm{H}_{2}$ density within the CS (5-4)-traced area for each clump, we need the corresponding kinetic temperature in addition to the $R_{(2-1) /(5-4)}$. When we took their $T_{\text {ex-clump }} \mathrm{s}$ as the corresponding kinetic temperatures in the CS (5-4)-traced areas (and this is in accordance with the LTE assumption which we would adopt in the following estimates), the clumps are marked on the maps as Figures 8 and 9 show. The corresponding average $\mathrm{H}_{2}$ densities of the clumps within the CS (5-4)traced areas, $n_{\mathrm{av}} \mathrm{s}$, are listed in Table 2 together with other parameters. The non-LTE analysis suggests that all these clumps have quite high $n_{\text {av }} \mathrm{s}$ in the CS (5-4)-traced areas. The non-LTE analysis results offer references for assuming densities in the subsequent estimates. We did not perform
non-LTE analysis for G333.1-0.4 since we only obtained CS (5-4) data.

\subsection{Clump Masses and $\mathrm{H}_{2}$ Column Densities}

To estimate the $\mathrm{H}_{2}$ column densities of these four clumps, we adopted the assumptions made in Li et al. (2007), namely,

(i) The medium along a certain line of sight has a single temperature (Goldsmith et al. 1997);

(ii) The absorption coefficient at $350 \mu \mathrm{m}, Q(350)$, is $2 \times 10^{-4}$;

(iii) The characteristic grain radius is $0.1 \mu \mathrm{m}$;

(iv) The grain density is $3 \mathrm{~g} \mathrm{~cm}^{-3}$;

(v) The gas to dust ratio (GDR) is 100 .

The clump mass can be expressed as

$$
\begin{aligned}
M_{\text {clump }}= & 0.10 M_{\odot}\left[\frac{2 \times 10^{-4}}{Q(350)}\right]\left[\frac{\lambda}{350 \mu \mathrm{m}}\right]^{3} \\
& {\left[\frac{D}{1 \mathrm{kpc}}\right]^{2}\left[\frac{\mathrm{GDR}}{100}\right]\left[\frac{S(\nu)}{\mathrm{Jy}}\right] P_{\mathrm{f}}\left(T_{\mathrm{d}}\right), }
\end{aligned}
$$

where $S(\nu)$ is the flux density of the cloud at $350 \mu \mathrm{m}$ at distance $D$, in Jy, $T_{\mathrm{d}}$ is the dust temperature, $P_{\mathrm{f}}\left(\mathrm{T}_{\mathrm{d}}\right)$ is the 


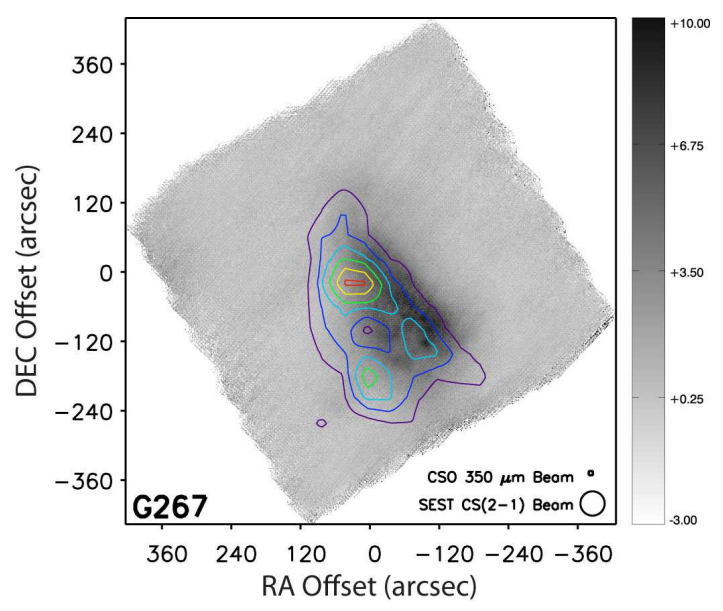

Fig. 5 The "hole" in G267.9-1.1. The background grey scale map is the $350 \mu \mathrm{m}$ emission map of G267.9-1.1 and the color contours show the CS (2-1) integrated intensity. The purple, dark blue, light blue, green, yellow and red contours represent $3,5,7,9,11$ and $13 \mathrm{~K} \mathrm{~km} \mathrm{~s}^{-1}$, respectively. The flux values on the color bar are in units of Jy beam ${ }^{-1}$.
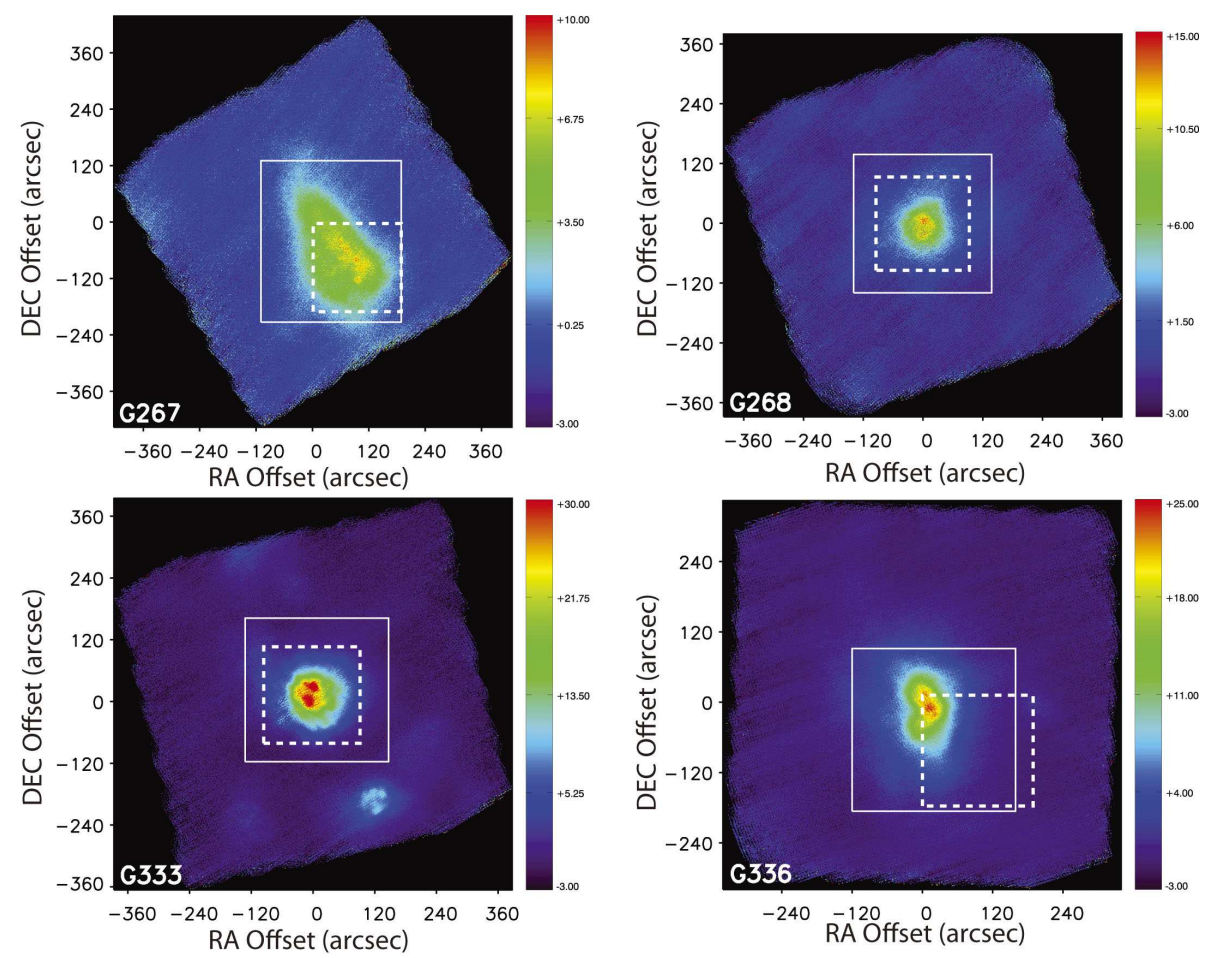

Fig. 6 The white boxes with solid lines and dashed lines represent the mass estimate area (also the CS (5-4)-traced area) and the ortho- $\mathrm{H}_{2} \mathrm{O}$ abundance estimate area, respectively. The flux values on the color bar are in units of Jy beam ${ }^{-1}$. Upper Left: G267.9-1.1 $350 \mu \mathrm{m}$ map. Upper Right: G268.4-0.9 $350 \mu \mathrm{m}$ map. Lower Left: G333.1-0.4 350 $\mu \mathrm{m}$ map. Lower Right: G336.5-1.5350 $\mu \mathrm{m}$ map.

Table 2 Non-LTE Analysis Parameters And Results

\begin{tabular}{lccccc}
\hline Clump & $R_{(2-1) /(5-4)}$ & $T_{\text {ex-clump }}(\mathrm{K})$ & $N_{\mathrm{CS}-\text { clump }}\left(\mathrm{cm}^{-2}\right)$ & $\begin{array}{c}n_{\mathrm{av}}\left(\mathrm{cm}^{-3}\right) \\
N_{\mathrm{CS}-\mathrm{clump}}=10^{13} \mathrm{~cm}^{-2}\end{array} N_{\mathrm{CS}-\mathrm{clump}_{\mathrm{av}}\left(\mathrm{cm}^{-3}\right)} 0^{14} \mathrm{~cm}^{-2}$ \\
\hline G267.9-1.1 & 1.0 & 22.4 & $5.1 \times 10^{13}$ & $2.0 \times 10^{6}$ & $2.9 \times 10^{6}$ \\
G268.4-0.9 & 2.5 & 11.5 & $6.8 \times 10^{13}$ & $3.8 \times 10^{6}$ & $-2 \times 10^{6}$ \\
G333.1-0.4 & - & - & - & - & - \\
G336.5-1.5 & 1.7 & 14.8 & $5.9 \times 10^{13}$ & $2.7 \times 10^{6}$ \\
\hline
\end{tabular}

Notes: ${ }^{a}$ We did not perform non-LTE analysis for clump G333.1-0.4 since there were only CS (5-4) data. 


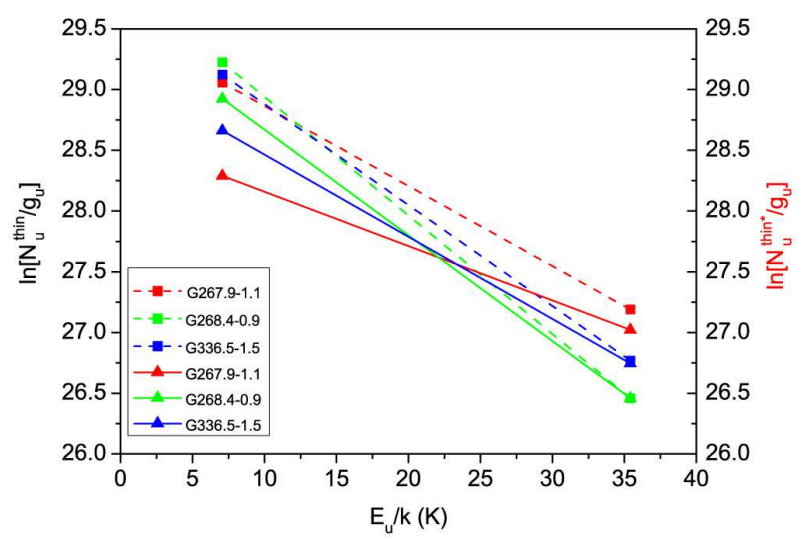

Fig. 7 Population diagrams for the "characteristic" CS excitation temperatures, $T_{\text {ex-clump }}$, of the CS (5-4)-traced areas in G267.9-1.1, G268.4-0.9 and G336.5-1.5. The plots in dashed lines with solid square symbols and in solid lines with solid triangle symbols were deduced from $N_{\mathrm{u}}^{\text {thin* }}$ s (Eq. (15)) and the last $N_{\mathrm{u}}^{\mathrm{thin}} \mathrm{s}$ (Eq. (16) ) retrieved in the iterations, respectively. The red, green and blue plots represent G267.9-1.1, G268.4-0.9 and G336.5-1.5, respectively.

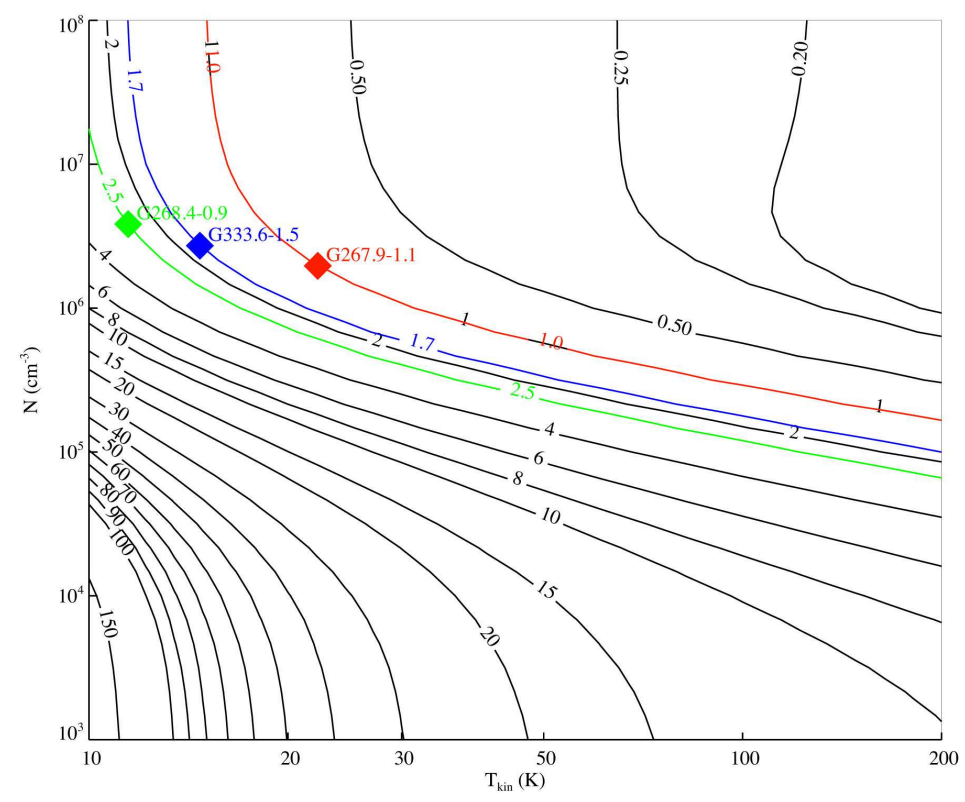

Fig. 8 Line intensity ratio map on the kinetic temperature-volume density plane with CS column densities of $10^{13} \mathrm{~cm}^{-2}$. The red, green and blue diamonds represent G267.9-1.1, G268.4-0.9 and G336.5-1.5 respectively.

Planck factor and

$$
P_{\mathrm{f}}\left(T_{\mathrm{d}}\right)=e^{h \nu / k T_{\mathrm{d}}}-1 .
$$

In this formula (Li et al. 2007), the dust temperature is an essential parameter. Although high density gases are present in these clumps, there can be a significant difference between the dust and gas temperatures at the same position (Goldsmith et al. 1997). However, when $n\left(\mathrm{H}_{2}\right)=$ $10^{6} \mathrm{~cm}^{-3}$, the dust temperature approximately equals the gas temperature (Li et al. 2007). Thus we can assume that along a certain line of sight the dust temperature equals the local gas temperature in this volume density condition. Since the CS (5-4) transition has a critical density of about $10^{6} \mathrm{~cm}^{-3}$, we assume that within the boundaries of CS (5-4)-traced areas $n\left(\mathrm{H}_{2}\right)=10^{6} \mathrm{~cm}^{-3}$. According to our non-LTE analysis with RADEX (in Section 3.2.2), this assumption is reasonable and we therefore adopt the approximation of dust temperature above in the following estimate.

Here we assume the gas temperature equals the kinetic temperature. Based on the relation between the CS excitation temperatures and the kinetic temperatures mentioned before, we actually adopted the calculated CS excitation temperatures as the local gas temperatures and the dust temperatures along the same lines of sight in the CS (54)-traced areas.

In the $350 \mu \mathrm{m}$ emission data, the grid spacing for the sampling is $1.5 \times 1.5 \operatorname{arcsec}^{2}$. By summing up the calculated mass of every cell in the sampling grid, we calcu- 


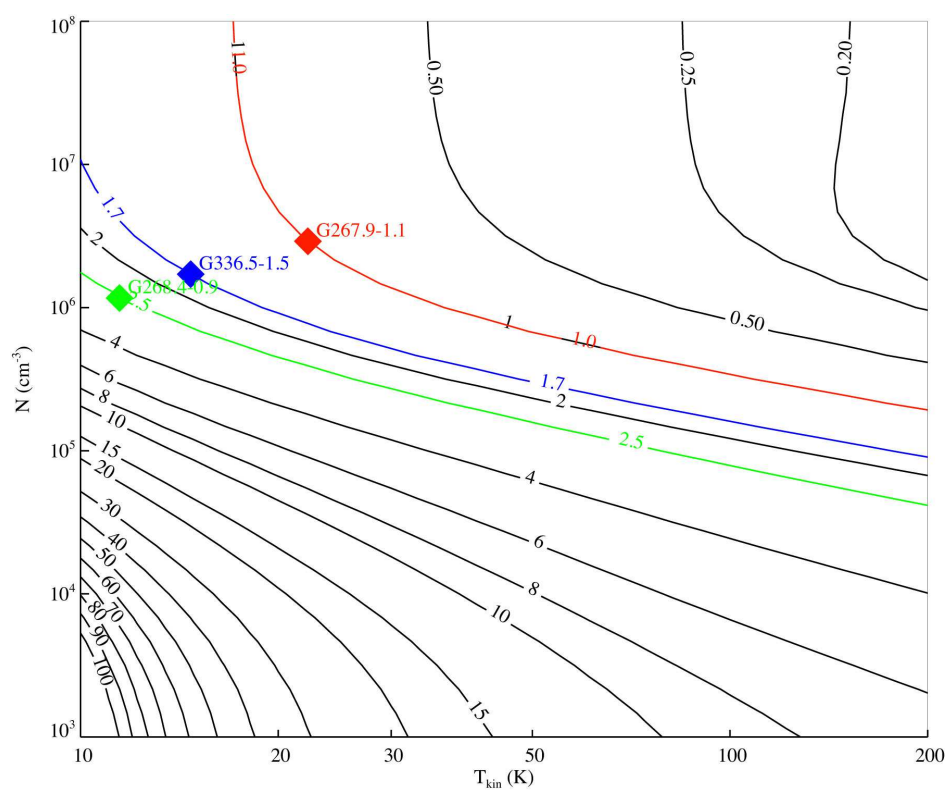

Fig. 9 Line intensity ratio map on the kinetic temperature-volume density plane with CS column densities of $10^{14} \mathrm{~cm}^{-2}$. The red, green and blue diamonds represent G267.9-1.1, G268.4-0.9 and G336.5-1.5 respectively.

lated the total mass of each clump. Subsequently we estimated the $\mathrm{H}_{2}$ column densities for each of the clumps cell by cell as

$$
N_{\mathrm{H}_{2}(\text { cell })}=M_{\text {clump }(\text { cell })} \frac{f}{(D \theta)^{2}},
$$

where $M_{\text {clump(cell) }}$ is the calculated mass of every single cell, $f$ is the mass fraction of $\mathrm{H}$ in gas with a ${ }^{4} \mathrm{He}$ to $\mathrm{H}$ ratio of 0.08459 (Balser 2006). $D$ is the distance from the clump, and $\theta$ is the sampling grid spacing, which is $1.5^{\prime \prime}$. Since the CS (5-4) line has a high critical density of about $10^{6} \mathrm{~cm}^{-3}$ and referencing our non-LTE analysis with RADEX (in Section 3.2.2) we assume that the element $\mathrm{H}$ is all in the form of $\mathrm{H}_{2}$ in the dense gases within the CS (5-4)-traced areas when we calculated the molecular hydrogen column densities. The calculated clump masses (of the CS (5-4)-traced areas, within the white boxes (solid lines) in Fig. 6) are listed in Column (6) of Table 3 The average $\mathrm{H}_{2}$ column densities in the areas used to estimate the ortho- $\mathrm{H}_{2} \mathrm{O}$ abundance (white boxes (dashed lines) in Fig. 6) are listed in Column (9) of Table 3 . The $\mathrm{H}_{2}$ column densities in these four clumps are on the order of magnitude $10^{22} \mathrm{~cm}^{-2}$ or $10^{23} \mathrm{~cm}^{-2}$.

\subsection{Ortho- $\mathrm{H}_{2} \mathrm{O}$ Abundance}

\subsubsection{Method}

The observation of the $557 \mathrm{GHz}$ ortho- $\mathrm{H}_{2} \mathrm{O} 1_{10}-1_{01}$ line was performed with SWAS with a pixel size of about

6 "The total mass" is the sum of the calculated mass of every single cell in each white box with solid lines in Figure 6 The boundaries of each box were determined based on the CS (5-4)-traced area, the area with available calculated CS excitation temperatures and the profile of the $350 \mu \mathrm{m}$ image (for G267.9-1.1).
$190 \times 190 \operatorname{arcsec}^{2}$ and a main beam efficiency of about 0.90 (Melnick 1995). This ground-state transition has a large spontaneous emission rate, $A$. As the stimulated absorption coefficient $B$ is proportional to the spontaneous emission rate, it leads to large opacities and makes excitation by photon trapping important (Wannier et al. 1991). Since the collisional de-excitation rate coefficient $C$ is far less than $A$, this line has a high critical density and the excitation is subthermal (in other words, the de-excitation of upper level molecules is dominated by emission photons rather than collisional de-excitation). Thus, although the line is expected to be optically thick at the line center even for a relatively low water abundance, every collisionally excited upper level molecule can always produce a photon which finally escapes the cloud (Snell et al. 2000a). Thus, the optically thick gas can be effectively thin (Snell et al. 2000a). Therefore, the integrated antenna temperature is proportional to the column density of ortho- $\mathrm{H}_{2} \mathrm{O}$ under known temperature and $\mathrm{H}_{2}$ volume density, according to Snell et al. (2000a),

$$
\int T_{\mathrm{b}} d v=C n_{\mathrm{H}_{2}} \frac{c^{3}}{2 \nu^{3} k} N\left(\mathrm{o}-\mathrm{H}_{2} \mathrm{O}\right) \frac{h \nu}{4 \pi} \exp \left(\frac{-h \nu}{k T_{\mathrm{k}}}\right) .
$$

$T_{\mathrm{b}} d v$ is the integrated intensity, in $\mathrm{K} \mathrm{km} \mathrm{s}^{-1} . C$ is the collisional de-excitation rate coefficient from level $1_{10}$ to level $1_{01}$.

\subsubsection{Kinetic temperature and other details}

Equation (20) can be written as

$$
X \cdot\left(\mathrm{o}-\mathrm{H}_{2} \mathrm{O}\right)=a \cdot \frac{\int T_{\mathrm{b}} d v}{N\left(H_{2}\right) n_{\mathrm{H}_{2}}},
$$


with

$$
a=\frac{1}{C \cdot \frac{c^{3}}{2 \nu^{3} k} \cdot \frac{h \nu}{4 \pi} \cdot \exp \left(\frac{-h \nu}{k T_{\mathrm{k}}}\right)}
$$

where $a$ is a constant at a given temperature.

We adopted an $\mathrm{H}_{2}$ volume density of $10^{6} \mathrm{~cm}^{-3}$ in the estimates since the areas where we estimated the ortho$\mathrm{H}_{2} \mathrm{O}$ abundances (the white boxes with dashed lines in Fig. 6) are in the CS (5-4)-traced areas. The average $\mathrm{H}_{2}$ column densities in the white boxes with dashed lines are listed in Column (9) of Table 3

The value of coefficient $a$ depends on the kinetic temperature and the corresponding $C$. Assuming that CS lines and the ortho- $\mathrm{H}_{2} \mathrm{O} 1_{10}-1_{01}$ line originate from the same gas, we take the calculated CS excitation temperatures as the kinetic temperature $T_{\mathrm{k}}$ at corresponding areas and adopt them in the estimate of ortho-water abundance. Since the pixel size of ortho- $\mathrm{H}_{2} \mathrm{O}$ data is much larger than that of the CS lines (the sampling spacing), there is an average effect for the kinetic temperature. We calculated the average $T_{\mathrm{k}}$ and the corresponding standard deviation (Table 3 Column (7) and (8)) to restrict the temperature range for the estimate. The collisional de-excitation rate coefficients were calculated according to the effective collisional excitation rate of ortho- $\mathrm{H}_{2} \mathrm{O}$ from level $1_{10}$ to level $1_{01}$ (hereafter the effective excitation rate) by para- or ortho- $\mathrm{H}_{2}$ and the ortho to para ratio of $\mathrm{H}_{2}$. The effective excitation rates were adopted from Dubernet et al. (2009) and Daniel et al. (2011) 7 from $5 \mathrm{~K}$ to $80 \mathrm{~K}$. Assuming that $\mathrm{H}_{2}$ molecules are in LTE, the ortho to para ratios of $\mathrm{H}_{2}$ were derived according to the $\mathrm{H}_{2}$ rotational energy levels from Dabrowski (1984) and the fractional population in the $\mathrm{H}_{2}$ rotational levels (Phillips et al. 1996).

\subsubsection{Ortho $-\mathrm{H}_{2} \mathrm{O}$ abundances}

The values of $a$ (see Eq. (21) and the estimated ortho- $\mathrm{H}_{2} \mathrm{O}$ abundances at the kinetic temperatures for every clump are listed in Table 4

The ortho- $\mathrm{H}_{2} \mathrm{O}$ abundances in the most probable temperature ranges of these four clumps (i.e., G267.91.1, 30-40 K; G268.4-0.9, 10-15 K; G333.1-0.4, 30$40 \mathrm{~K}$; G336.5-1.5, 15-20 K. The corresponding ortho$\mathrm{H}_{2} \mathrm{O}$ abundances were called "the typical ortho- $\mathrm{H}_{2} \mathrm{O}$ abundances" in Section 4.1) are presented in Figure 10 together with some other ortho- $\mathrm{H}_{2} \mathrm{O}$ water abundances of giant molecular cloud (GMC) cores (Snell et al. 2000b) and molecular outflows (Franklin et al. 2008), which are based on the same ortho- $\mathrm{H}_{2} \mathrm{O}$ transition observed by SWAS. The ortho- $\mathrm{H}_{2} \mathrm{O}$ abundances of these four clumps are at a low level compared with other results.

\section{5 $\mathrm{N}_{2} \mathrm{H}^{+}$Abundances}

Since the critical density of the $\mathrm{N}_{2} \mathrm{H}^{+}$(1-0) line is far lower than that of the CS (5-4) line, we can assume that

\footnotetext{
7 i.e., the "effective rate coefficient" in these two papers
}

CS lines ((5-4) and (2-1)) and the $\mathrm{N}_{2} \mathrm{H}^{+}$(1-0) line are all thermally populated in the CS (5-4)-traced areas, thus their excitation temperatures are all approximately equal to the kinetic temperatures. In this situation, we can adopt the excitation temperatures of CS as the excitation temperatures of $\mathrm{N}_{2} \mathrm{H}^{+}$at the same positions. Then with the same assumptions and approximations we used in calculation of the column density of upper level CS molecules, we calculate the corrected $\mathrm{N}_{2} \mathrm{H}^{+}$column density at $J=1$. The $\mathrm{N}_{2} \mathrm{H}^{+}$column density $N_{\text {total }}$ is estimated as

$$
N_{\text {total }}=N_{\mathrm{J}=1} \frac{Z}{2 J+1} \exp \left[\frac{h B_{\mathrm{e}} J(J+1)}{k T}\right],
$$

according to Rohlfs \& Wilson (1996). $N_{\mathrm{J}=1}$ is the $\mathrm{N}_{2} \mathrm{H}^{+}$ column density without the Rayleigh-Jeans approximation at $J=1 . B_{\mathrm{e}}$ is the rotational constant of the $\mathrm{N}_{2} \mathrm{H}^{+}$ molecule at vibrational energy level $v=08 . J$ is the rotational quantum number of the upper level and $J=1 . k$ is the Boltzmann constant and $h$ is the Planck constant. We adopt the excitation temperatures of CS as the temperature T. $Z$ is the rotational partition function of $\mathrm{N}_{2} \mathrm{H}^{+}$. Since $\mathrm{N}_{2} \mathrm{H}^{+}$is a linear molecule (Mangum \& Shirley 2015), when the contribution of the vibrational excited states is not taken into account,

$$
Z \simeq \sum_{J=0}^{\infty}(2 J+1) \exp \left(-\frac{h B_{0} J(J+1)}{k T}\right) .
$$

$B_{0}$ is the rigid rotor rotational constant of the $\mathrm{N}_{2} \mathrm{H}^{+}$ molecule at the ground vibrational state $v=0$ and $B_{0}=$ $46586.88 \mathrm{MHz}$ (Mangum \& Shirley 2015). $J$ is the rotational quantum number and $J=0,1,2, \ldots, k . h$ and $T$ are as the same as in Equation (23).

According to Mangum \& Shirley (2015), if we use one or several hyperfine transition(s) that can be observed to derive the column density of $\mathrm{N}_{2} \mathrm{H}^{+}$, we must take the relative line strengths of the hyperfine transition(s) into consideration. However, in our observation the spectra cover all the seven hyperfine transitions which can be observed in the $J=1 \rightarrow 0$ transition. Thus, we just calculate the rotational partition functions at corresponding temperatures and then estimate the $\mathrm{N}_{2} \mathrm{H}^{+}$column densities. We average the estimated $\mathrm{H}_{2}$ column densities at every $\mathrm{N}_{2} \mathrm{H}^{+}$pixel and then estimate the $\mathrm{N}_{2} \mathrm{H}^{+}$abundances. The results are shown in Table 5 and Figure 11 and all offsets are relative to the corresponding coordinates (J2000) in Table 3 and the unit is arcsec.

\section{DISCUSSION}

\subsection{Ortho- $\mathrm{H}_{2} \mathrm{O}$ Abundances}

The typical ortho- $\mathrm{H}_{2} \mathrm{O}$ abundances of these four clumps are in the range $3.7 \times 10^{-10}-5.8 \times 10^{-10}$ for G267.9$1.1,4.1 \times 10^{-10}-4.2 \times 10^{-10}$ for G268.4-0.9, $3.8 \times 10^{-10}$

\footnotetext{
8 http://www.cv.nrao.edu/php/splat/species_metadata_displayer.php? species_id $=148$
} 
Table 3 The Parameters of the Four

Clumps

\begin{tabular}{lcccccccc}
\hline Source & RA J2000 & Dec J2000 & $\begin{array}{c}\text { Distance } \\
(\mathrm{kpc})\end{array}$ & $\begin{array}{c}\text { Average } T_{\mathrm{ex}} \\
(\mathrm{K})\end{array}$ & $\begin{array}{c}\text { Mass } \\
\left(M_{\odot}\right)\end{array}$ & $\begin{array}{c}\text { Average } T_{\mathrm{k}} \\
(\mathrm{K})\end{array}$ & $\begin{array}{c}\mathrm{SD}^{f} \text { of } T_{\mathrm{k}} \\
(\mathrm{K})\end{array}$ & $\begin{array}{c}\text { Average } N\left(\mathrm{H}_{2}\right) \\
\left(\mathrm{cm}^{-2}\right) \\
(1)\end{array}$ \\
$(2)$ & $(3)$ & $(4)$ & $(5)$ & $(6)$ & $(7)$ & $(8)$ & $3.7 \times 10^{22}$ \\
\hline G267.9-1.1 & $08: 59: 12.00$ & $-47: 29: 04.0$ & $1.5^{a}$ & 26.5 & $2.5 \times 10^{3}$ & 31.1 & 30.7 & 3.7 \\
G268.4-0.9 & $09: 01: 54.30$ & $-47: 43: 59.0$ & $1.3^{b}$ & 11.5 & $4.6 \times 10^{3}$ & 11.5 & 1.4 & $1.2 \times 10^{23}$ \\
G333.1-0.4 & $16: 21: 02.10$ & $-50: 35: 15.0$ & $3.6^{c}$ & $-e$ & $1.2 \times 10^{4}$ & 31.9 & - & $4.4 \times 10^{22}$ \\
G336.5-1.5 & $16: 40: 00.20$ & $-48: 51: 20.0$ & $1.4^{d}$ & 16.2 & $9.0 \times 10^{3}$ & 17.8 & 6.3 & $8.5 \times 10^{22}$ \\
\hline
\end{tabular}

Notes: ${ }^{a}$ Frogel \& Persson 1974. ${ }^{b}$ Zinchenko et al. 1995. ${ }^{c}$ Lockman 1979. ${ }^{d}$ Thompson et al. 2004. ${ }^{e}$ We could not perform the estimate of CS excitation temperatures for clump G333.1-0.4 since there were only CS (5-4) data. We adopted its kinetic temperature $(31.9 \mathrm{~K})$ from Lowe et al. (2014) and applied it in the following estimates. This kinetic temperature was derived from the rotation temperature of the $\mathrm{NH}_{3}(1,1)$ and $(2,2)$ transitions. " $f$ D" stands for "standard deviation."

Table 4 The Values of coefficient $a$ and Ortho- $\mathrm{H}_{2} \mathrm{O}$

Abundances

\begin{tabular}{lccccc}
\hline$T_{\mathrm{k}}(\mathrm{K})$ & $a$ & $\begin{array}{c}\mathrm{G} 267.9-1.1 \\
\left(\times 10^{-10}\right)\end{array}$ & $\begin{array}{c}\text { G268.4-0.9 } \\
\left(\times 10^{-10}\right)\end{array}$ & $\begin{array}{c}\text { G333.1-0.4 } \\
\left(\times 10^{-10}\right)\end{array}$ & $\begin{array}{c}\text { G336.5-1.5 } \\
\left(\times 10^{-10}\right)\end{array}$ \\
\hline 5 & $3.7 \times 10^{21}$ & 7.1 & - & - & - \\
10 & $2.6 \times 10^{20}$ & 7.0 & 4.1 & - & 5.7 \\
15 & $1.1 \times 10^{20}$ & 7.1 & 4.2 & - & 5.7 \\
20 & $6.7 \times 10^{19}$ & 7.0 & - & 7.2 & 5.7 \\
30 & $3.6 \times 10^{19}$ & 5.8 & - & 5.9 & 4.7 \\
40 & $1.8 \times 10^{19}$ & 3.7 & - & 3.8 & - \\
50 & $1.0 \times 10^{19}$ & 2.4 & - & - & - \\
60 & $7.2 \times 10^{18}$ & 1.8 & - & - & - \\
80 & $4.7 \times 10^{18}$ & 1.3 & - & - & - \\
\hline
\end{tabular}

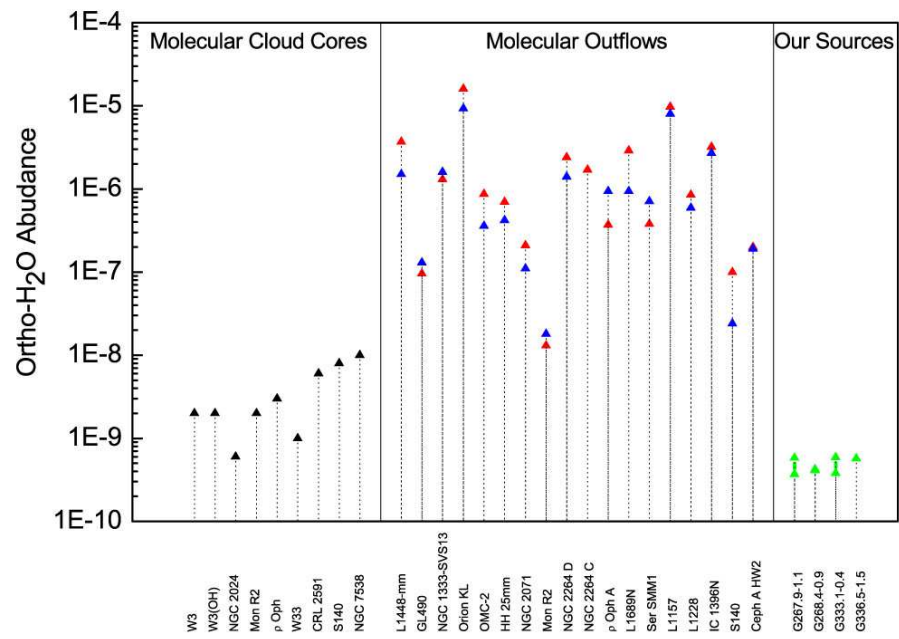

Fig. 10 The estimated ortho- $\mathrm{H}_{2} \mathrm{O}$ abundances of clump G267.9-1.1, G268.4-0.9, G333.1-0.4 and G336.5-1.5 in the most probable temperature ranges (G267.9-1.1, 30-40 K; G268.4-0.9, 10-15 K; G333.1-0.4, 30-40 K; G336.5-1.5, 15-20 K), in comparison with other results derived from the $557 \mathrm{GHz}$ ortho- $\mathrm{H}_{2} \mathrm{O} 1_{10}-1_{01}$ line observed by SWAS. The solid black triangles represent the molecular cloud cores from Snell et al. (2000b). The solid red triangles and solid blue triangles represent the redshifted and blueshifted emission respectively in each molecular outflow from Franklin et al. (2008). The solid green triangles linked with green lines show the ortho- $\mathrm{H}_{2} \mathrm{O}$ abundance ranges of the four clumps in this paper.

Table $5 \mathrm{~N}_{2} \mathrm{H}^{+}$Abundances

\begin{tabular}{ll}
\hline Clump & $\mathrm{N}_{2} \mathrm{H}^{+}$Abundances \\
\hline G267.9-1.1 & $1.0 \times 10^{-10}-1.5 \times 10^{-8}$ \\
G268.4-0.9 & $6.1 \times 10^{-11}-4.3 \times 10^{-9}$ \\
G333.1-0.4 & $2.6 \times 10^{-10}-4.2 \times 10^{-9}$ \\
G336.5-1.5 & $5.6 \times 10^{-11}-1.4 \times 10^{-9}$ \\
\hline
\end{tabular}



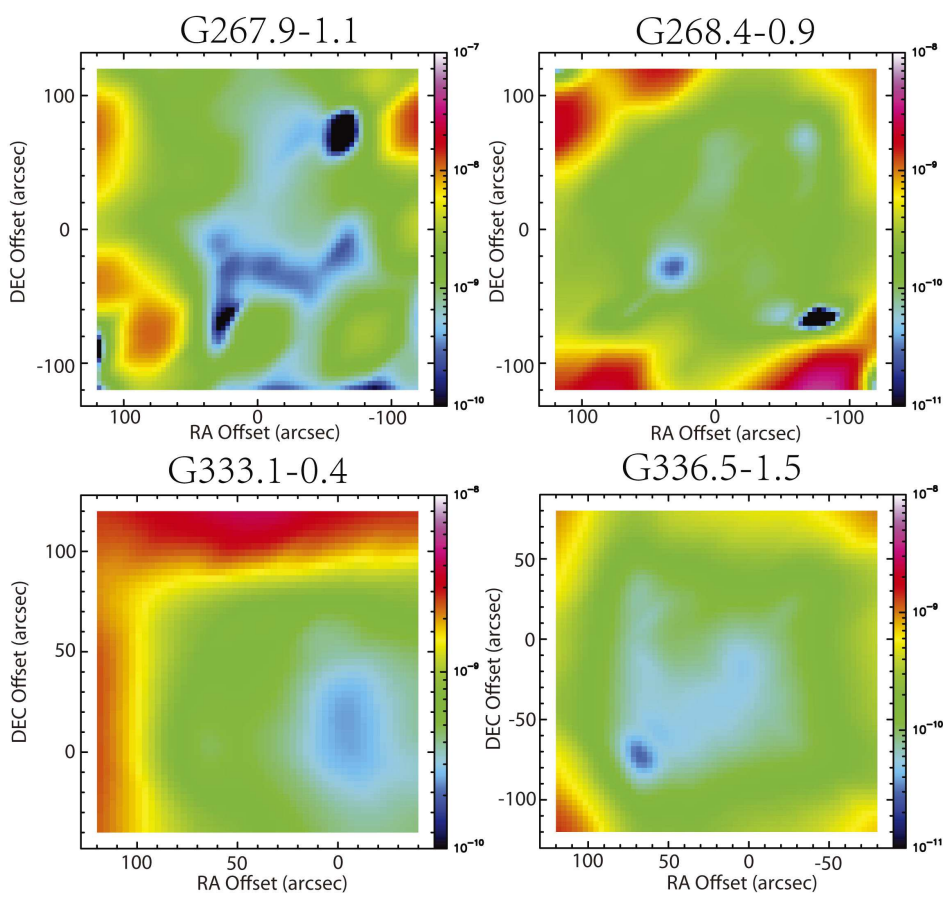

Fig. 11 Upper left: G267.9-1.1 $\mathrm{N}_{2} \mathrm{H}^{+}$abundance. Upper right: G268.4-0.9 $\mathrm{N}_{2} \mathrm{H}^{+}$abundance. Lower left: G333.1-0.4 $\mathrm{N}_{2} \mathrm{H}^{+}$abundance. Lower right: G336.5-1.5 $\mathrm{N}_{2} \mathrm{H}^{+}$abundance. The color bars are abundance scales.

$-5.9 \times 10^{-10}$ for G333.1-0.4 and around $5.7 \times 10^{-10}$ for G336.5-1.5.

The typical ortho- $\mathrm{H}_{2} \mathrm{O}$ abundances are at a low level compared with those of cold $(T<50 \mathrm{~K})$ GMC cores estimated with the same principle by Snell et al. (2000b). The upper limits of the ortho- $\mathrm{H}_{2} \mathrm{O}$ abundances of these four clumps are on the order of $10^{-10}$, lower than the abundances of most of the GMC cores in Snell et al. (2000b). In our estimate, the effective excitation rates we adopted for para- $\mathrm{H}_{2}(j=0)$ are larger than those from Phillips et al. (1996) by a factor of $\sim 1-3$ at temperatures from $20 \mathrm{~K}$ to $80 \mathrm{~K}$. This fact should be noticed when comparing our results with the results in Snell et al. (2000b) or Franklin et al. (2008) (see Fig.10).

The low abundances may be caused by the low temperatures of these clumps if we consider the water vapor originating from the interior of the clumps. Even at temperatures as low as $10 \mathrm{~K}$, water can form in the ISM van Dishoeck et al. 2013). However, at such low temperatures and high densities, the freeze-out procedure dominates (Bergin \& van Dishoeck 2012) and until the temperature is above about $100 \mathrm{~K}$ (Hollenbach et al. 2009), water molecules can be desorbed through thermal sublimation. Also, in the interior of the dense clump, the desorption of frozen water molecules is unlikely to be caused by photodesorption. Although there is an average effect for the temperature in the large SWAS beam (and in the ortho$\mathrm{H}_{2} \mathrm{O}$ pixels, also), we can find that the areas along the line of sight from where the ortho- $\mathrm{H}_{2} \mathrm{O}$ emissions originate are quite cold, or in other words, are not warm enough to produce much water vapor. Consequently the ortho- $\mathrm{H}_{2} \mathrm{O}$ abundances are low.

On the other hand, since these clumps are all located in star forming regions, the ortho- $\mathrm{H}_{2} \mathrm{O}$ emission therefore can originate primarily at the intermediate depth of these clumps where neither the photodissociation nor the freezing out of the $\mathrm{H}_{2} \mathrm{O}$ molecules, but the photodesorption process dominates according to a model for the temperature and chemical structure in molecular clouds (Hollenbach et al. 2009). Thus, since we took the $\mathrm{H}_{2}$ column densities along the line of sight to estimate the abundances, it consequently results in apparent low ortho- $\mathrm{H}_{2} \mathrm{O}$ abundance for the whole clump while in the photodesorbed layer the water vapor is actually more abundant.

In addition, there are also considerations of possible factors which can cause the apparent low ortho- $\mathrm{H}_{2} \mathrm{O}$ abundances of those clumps but have been masked due to the averaging effect of the large SWAS beam. For example, small structures in the clump such as a hot outflow may contribute the majority of the observed gaseous water. The water abundance may vary greatly within the same clump. A similar phenomenon has been confirmed in the outflow powered by L1157-mm (a low-mass Class 0 protostar), in which the water abundance of the hot component is about two orders of magnitude higher than that of the nearby colder component (Busquet et al. 2014). In the areas we estimated ortho-water abundance, the maximum CS excitation temperatures of G267.9-1.1, G268.40.9 and G336.5-1.5 are $165.2 \mathrm{~K}$ (and the second largest value is $86.6 \mathrm{~K}$ ), $15.8 \mathrm{~K}$ and $42.3 \mathrm{~K}$, respectively (and for $\mathrm{G} 333.1-0.4$, the average kinetic temperature is $31.9 \mathrm{~K}$, ac- 
cording to Lowe et al. (2014)). For G267.9-1.1, there is a possibility that the warm component makes a big contribution to the origination of gaseous water. However, we cannot infer more information on structures smaller than the SWAS beam size which can further reveal the origination of gaseous water.

If the ortho- $\mathrm{H}_{2} \mathrm{O} 1_{10}-1_{01}$ line originates from the same gas as CS lines, as we assumed when we estimated the ortho-water abundance, then the CS lines may help to find traces of outflows. The CS spectra of G267.9-1.1 have broad wings. We found that $267.9-1.1$ as well as G268.4-0.9 does show velocity variation over the clump on its channel map, but the spatial resolution of CS data is not high enough to identify the outflows. Lapinov et al. (1998) have mapped G268.4-0.9 (G268.42-0.85) in CS $J=5-4$ (SEST, $20^{\prime \prime}$ sampling spacing) and $J=7-6$ (the CSO telescope, $10^{\prime \prime}$ sampling spacing) lines. They used the Maximum Entropy Method (MEM) deconvolution technique to achieve higher angular resolution (Lapinov et al. 1998). In their study, the CS (5-4) map shows two peaks with an LSR velocity difference of about $0.7 \mathrm{Km} \mathrm{s}^{-1}$ and on the CS (7-6) map, a bipolar structure was identified but no further temperature information was offered for this bipolar structure (Lapinov et al. 1998).

To be honest and objective, it is kind of arbitrary to assume such a high density over the whole area in which we estimated the ortho- $\mathrm{H}_{2} \mathrm{O}$ abundance in each clump. There are very likely to be $\mathrm{H}_{2}$ density gradients in these areas. If we adopt $10^{4} \mathrm{~cm}^{-3}$ rather than $10^{6} \mathrm{~cm}^{-3}$ as the $\mathrm{H}_{2}$ density in the ortho- $\mathrm{H}_{2} \mathrm{O}$ abundance estimation, then the typical ortho- $\mathrm{H}_{2} \mathrm{O}$ abundances will be at the magnitude of $10^{-8}$, the same as those of most of the GMC cores in Snell et al. (2000b).

\section{2 $\mathrm{N}_{2} \mathrm{H}^{+}$Abundances}

The $\mathrm{N}_{2} \mathrm{H}^{+}$abundances of these four clumps are in the range of $1.0 \times 10^{-10}-1.5 \times 10^{-8}$ for G267.9-1.1, $6.1 \times 10^{-11}-4.3 \times 10^{-9}$ for G268.4-0.9, $2.6 \times 10^{-10}$ $-4.2 \times 10^{-9}$ for G333.1-0.4 and $5.6 \times 10^{-11}-1.4 \times$ $10^{-9}$ for G336.5-1.5. The distribution of $\mathrm{N}_{2} \mathrm{H}^{+}$abundance in each clump has a common decreasing trend toward the center. Although the abundance distributions we derived are only projected results in a plane perpendicular to the line of sight, we noticed that Melnick et al. (2011) suggested that $\mathrm{N}_{2} \mathrm{H}^{+}$is likely to be distributed primarily in the clump rather than in the surface layers. When it comes to the depletion of $\mathrm{N}_{2} \mathrm{H}^{+}, \mathrm{CO}$ and electrons are the major destroyers of $\mathrm{N}_{2} \mathrm{H}^{+}$in the gas phase and their reaction with $\mathrm{N}_{2} \mathrm{H}^{+}$generates $\mathrm{N}_{2}$ (Aikawa et al. 2001, Aikawa et al. 2005). According to Bergin \& Tafalla (2007), in the dense cores the neutrals (including CO) will rapidly freeze onto the grains. Consequently, the abundance of $\mathrm{N}_{2} \mathrm{H}^{+}$will increase as a result of the disappearance of CO (Bergin \& Tafalla 2007). However, when we focus on temperature, we notice that when the dust temperature rises from $10 \mathrm{~K}$ to about $30 \mathrm{~K}$, $\mathrm{CO}$ begins to sub- limate (van Dishoeck et al. 2013). Thus, based on the gas temperatures we estimated (the dust temperatures are approximately equal to local gas temperatures at such a high density as we had assumed), we can infer that in the high density center of the clump, the gas and dust are warm enough and the gaseous $\mathrm{CO}$ is abundant. $\mathrm{N}_{2} \mathrm{H}^{+}$is therefore depleted by $\mathrm{CO}$ and that leads to a drop in $\mathrm{N}_{2} \mathrm{H}^{+}$ abundance toward the center.

\section{CONCLUSIONS}

We studied G267.9-1.1, G268.4-0.9, G333.1-0.4 and G336.5-1.5, four of the brightest ortho- $\mathrm{H}_{2} \mathrm{O}$ sources in the southern sky observed by SWAS. We estimated their CS excitation temperatures in the CS (5-4)-traced areas and estimated their masses. Based on the temperatures and the masses, we then derived their average $\mathrm{H}_{2}$ column densities and estimated the ortho- $\mathrm{H}_{2} \mathrm{O}$ and $\mathrm{N}_{2} \mathrm{H}^{+}$abundances.

The typical molecular clumps in our study have $\mathrm{H}_{2}$ column densities of $\sim 10^{22}$ to $10^{23} \mathrm{~cm}^{-2}$ and ortho- $\mathrm{H}_{2} \mathrm{O}$ abundances of $10^{-10}$. The low ortho- $\mathrm{H}_{2} \mathrm{O}$ abundances can be caused by the freeze-out of $\mathrm{H}_{2} \mathrm{O}$ in the interior of the clumps due to the low temperatures if the ortho- $\mathrm{H}_{2} \mathrm{O}$ originates from the interior of the clumps.

The typical $\mathrm{N}_{2} \mathrm{H}^{+}$abundances of these four clumps in this paper range from $10^{-11}$ to $10^{-9}$. Since in the center areas of the clumps, dust at such a high density is at temperatures such that $\mathrm{CO}$ can be released into the gas phase, the common trend of abundance decreasing toward the center of the clump can be a result of the depletion of $\mathrm{N}_{2} \mathrm{H}^{+}$ caused by $\mathrm{CO}$.

Acknowledgements We sincerely thank the anonymous referee and the scientific editor for their wholehearted and patient help and the valuable advice they provided to help us improve this paper. This research is supported by the National Basic Research Program of China (973 program, Nos. 2012CB821800 and 2015CB857100), the National Natural Science Foundation of China (No. 11373038) and the Strategic Priority Research Program "The Emergence of Cosmological Structures" of the Chinese Academy of Sciences (Grant No. XDB09000000).

\section{Appendix A: SUPPLEMENTARY MATERIAL}

In the temperature range $(20 \mathrm{~K}$ to $80 \mathrm{~K})$, para- and ortho$\mathrm{H}_{2}$ are barely populated at energy levels other than $j_{2}=0$ and $j_{2}=1\left(j_{2}\right.$ is the rotational level of $\left.\mathrm{H}_{2}\right)$, respectively. We adopted the effective rate coefficients at $j_{2}=0$ and $j_{2}$ $=1$ from Dubernet et al. (2009) and Daniel et al. (2011).

For G267.9-1.1 and G336.5-1.5, we estimated the ortho- $\mathrm{H}_{2} \mathrm{O}$ abundance at the ortho- $\mathrm{H}_{2} \mathrm{O}$ integrated intensity maximum pixel in FITS format data. Although there is more than one sampling cell having emission in the CLASS format data, when written in FITS format, only the pixel with maximum integrated intensity $\left(190 \times 190 \operatorname{arcsec}^{2}\right.$, the boxes with white dashed lines in 
Fig. 6) are located within the areas with calculated $\mathrm{H}_{2}$ column densities. However, the estimated ortho- $\mathrm{H}_{2} \mathrm{O}$ abundance of the pixels with maximum integrated intensity still characterize the ortho- $\mathrm{H}_{2} \mathrm{O}$ abundance of these clump in a sense. For G268.4-0.9 and G333.1-0.4, the CLASS format ortho- $\mathrm{H}_{2} \mathrm{O}$ data only have one sampling cell and the center of the cell is the same as the corresponding coordinates in Table 3 (with a deviation of $0.01 \mathrm{~s}$ on RA). So we just estimated the ortho- $\mathrm{H}_{2} \mathrm{O}$ abundance in the only sampling cell (the box with white dashed lines in Fig. 6 $190 \times 190 \operatorname{arcsec}^{2}$, the same as the pixel size of the FITS format data).

We calculate the ortho- $\mathrm{H}_{2} \mathrm{O}$ abundance of G336.5-1.5 in the overlapping area of the white box (dashed lines) and the white box (solid lines) with corresponding average $T_{\mathrm{k}}$, average $\mathrm{H}_{2}$ column density and proportionally corrected ortho- $\mathrm{H}_{2} \mathrm{O}$ integrated intensity.

When calculating the partition functions of CS and $\mathrm{N}_{2} \mathrm{H}^{+}$, we summed the polynomial term by term from the $J=0$ level, following the incremental rotational quantum number. When the value of a term is less than $0.1 \%$ of the sum of all the terms of the lower levels, then this term will be the last term added in the summation.

\section{References}

Aikawa, Y., Ohashi, N., Inutsuka, S.-i., Herbst, E., \& Takakuwa, S. 2001, ApJ, 552, 639

Aikawa, Y., Herbst, E., Roberts, H., \& Caselli, P. 2005, ApJ, 620, 330

Ashby, M. L. N., Bergin, E. A., Plume, R., et al. 2000, ApJ, 539, L115

Balser, D. S. 2006, AJ, 132, 2326

Beard, M. 1966, Australian Journal of Physics, 19, 141

Bergin, E. A., \& Tafalla, M. 2007, ARA\&A, 45, 339

Bergin, E. A., \& van Dishoeck, E. F. 2012, Philosophical Transactions of the Royal Society of London Series A, 370, 2778

Braz, M. A., Gregorio Hetem, J. C., Scalise, Jr., E., Monteiro Do Vale, J. L., \& Gaylard, M. 1989, A\&AS, 77, 465

Busquet, G., Lefloch, B., Benedettini, M., et al. 2014, A\&A, 561, A120

Caswell, J. L., Batchelor, R. A., Haynes, R. F., \& Huchtmeier, W. K. 1974, Australian Journal of Physics, 27, 417

Cheung, A. C., Rank, D. M., \& Townes, C. H. 1969, Nature, 221, 917

Dabrowski, I. 1984, Canadian Journal of Physics, 62, 1639, in Ro-vibrational Collisional Excitation Database: BASECOL http://basecol.obspm.fr/

Daniel, F., Dubernet, M.-L., \& Grosjean, A. 2011, A\&A, 536, A76, in Ro-vibrational Collisional Excitation Database: BASECOL http://basecol.obspm.fr/

Doty, S. D., \& Neufeld, D. A. 1997, ApJ, 489, 122

Dowell, C. D., Allen, C. A., Babu, R. S., et al. 2003, in Society of Photo-Optical Instrumentation Engineers (SPIE) Conference
Series, 4855, Millimeter and Submillimeter Detectors for Astronomy, eds. T. G. Phillips, \& J. Zmuidzinas, 73

Dubernet, M.-L., Daniel, F., Grosjean, A., \& Lin, C. Y. 2009, A\&A, 497, 911, in Ro-vibrational Collisional Excitation Database: BASECOL http://basecol.obspm.fr/

Emprechtinger, M., Lis, D. C., Bell, T., et al. 2010, A\&A, 521, L28

Franklin, J., Snell, R. L., Kaufman, M. J., et al. 2008, ApJ, 674, 1015

Frogel, J. A., \& Persson, S. E. 1974, ApJ, 192, 351

Goldsmith, P. F., Bergin, E. A., \& Lis, D. C. 1997, ApJ, 491, 615

Goldsmith, P. F., \& Langer, W. D. 1999, ApJ, 517, 209

Goss, W. M., \& Shaver, P. A. 1970, Australian Journal of Physics Astrophysical Supplement, 14, 1

Hollenbach, D., Kaufman, M. J., Bergin, E. A., \& Melnick, G. J. 2009, ApJ, 690, 1497 
Juvela, M. 1996, A\&AS, 118, 191

Kaufmann, P., Gammon, R. H., Ibanez, A. L., et al. 1976, Nature, 260, 306

Kewley, R., Sastry, K. V. L. N., Winnewisser, M., \& Gordy, W. 1963, J. Chem. Phys., 39, 2856

Kristensen, L. E., \& van Dishoeck, E. F. 2011, Astronomische Nachrichten, 332, 475

Kristensen, L. E., van Dishoeck, E. F., Bergin, E. A., et al. 2012, A\&A, 542, A8

Lapinov, A. V., Schilke, P., Juvela, M., \& Zinchenko, I. I. 1998, A\&A, 336, 1007

Li, D., González-Alfonso, E., \& Melnick, G. 2004, in Bulletin of the American Astronomical Society, 36, American Astronomical Society Meeting Abstracts \#204, 985

Li, D., Velusamy, T., Goldsmith, P. F., \& Langer, W. D. 2007, ApJ, 655, 351

Lockman, F. J. 1979, ApJ, 232, 761

Lowe, V., Cunningham, M. R., Urquhart, J. S., et al. 2014, MNRAS, 441, 256

Manchester, B. A., \& Goss, W. M. 1969, Australian Journal of Physics Astrophysical Supplement, 11, 35

Mangum, J. G., \& Shirley, Y. L. 2015, PASP, 127, 266

Mardones, D., Myers, P. C., Tafalla, M., et al. 1997, ApJ, 489, 719

Melnick, G. J. 1995, in Astronomical Society of the Pacific Conference Series, Vol. 73, From Gas to Stars to Dust, eds. M. R. Haas, J. A. Davidson, \& E. F. Erickson, 673

Melnick, G. J., Stauffer, J. R., Ashby, M. L. N., et al. 2000, ApJ, 539, L77

Melnick, G. J., Tolls, V., Snell, R. L., et al. 2011, ApJ, 727, 13

Phillips, T. R., Maluendes, S., \& Green, S. 1996, ApJS, 107, 467

Rohlfs, K., \& Wilson, T. L. 1996, Tools of Radio Astronomy (Springer-Verlag Berlin Heidelberg New York)

Shaver, P. A., \& Goss, W. M. 1970, Australian Journal of Physics Astrophysical Supplement, 14, 133

Snell, R. L., Howe, J. E., Ashby, M. L. N., et al. 2000a, ApJ, 539, L93

Snell, R. L., Howe, J. E., Ashby, M. L. N., et al. 2000b, ApJ, 539, L101

Sugitani, K., \& Ogura, K. 1994, ApJS, 92, 163

Thompson, M. A., Urquhart, J. S., \& White, G. J. 2004, A\&A, 415,627

Valdettaro, R., Chapman, J. M., Lovell, J. E. J., \& Palla, F. 2007, A\&A, 466, 247

van der Tak, F. F. S., Black, J. H., Schöier, F. L., Jansen, D. J., \& van Dishoeck, E. F. 2007, A\&A, 468, 627

van Dishoeck, E. F., Herbst, E., \& Neufeld, D. A. 2013, Chemical Reviews, 113, 9043

Wannier, P. G., Pagani, L., Kuiper, T. B. H., et al. 1991, ApJ, 377, 171

Wilson, T. L., Mezger, P. G., Gardner, F. F., \& Milne, D. K. 1970, A\&A, 6, 364

Yamaguchi, R., Saito, H., Mizuno, N., et al. 1999, PASJ, 51, 791

Zinchenko, I., Mattila, K., \& Toriseva, M. 1995, A\&AS, 111, 95 